Article

\title{
Investigation into Pump Mode Flow Dynamics for a Mixed Flow PAT with Adjustable Runner Blades
}

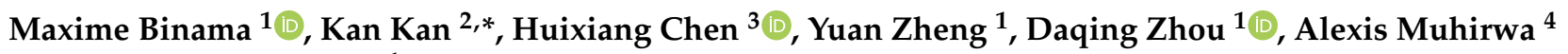 \\ and Godfrey M. Bwimba ${ }^{1}$ \\ 1 College of Water Conservancy and Hydropower Engineering, Hohai University, Nanjing 210098, China; \\ binamamaxime@hhu.edu.cn (M.B.); zhengyuan@hhu.edu.cn (Y.Z.); zhoudaqing@hhu.edu.cn (D.Z.); \\ gbwimba@gmail.com (G.M.B.) \\ 2 College of Energy and Electrical Engineering, Hohai University, Nanjing 210098, China \\ 3 College of Agricultural Engineering, Hohai University, Nanjing 210098, China; chenhuixiang@hhu.edu.cn \\ 4 School of Energy Science and Engineering, Harbin Institute of Technology, Harbin 150001, China; \\ muhalexis80@yahoo.co.uk \\ * Correspondence: kankan@hhu.edu.cn
}

Citation: Binama, M.; Kan, K.; Chen, H.; Zheng, Y.; Zhou, D.; Muhirwa, A.; Bwimba, G.M. Investigation into Pump Mode Flow Dynamics for a Mixed Flow PAT with Adjustable Runner Blades. Energies 2021, 14, 2690. https://doi.org/10.3390/en14092690

Academic Editor: Satoru Okamoto

Received: 29 March 2021

Accepted: 4 May 2021

Published: 7 May 2021

Publisher's Note: MDPI stays neutral with regard to jurisdictional claims in published maps and institutional affiliations.

Copyright: (c) 2021 by the authors. Licensee MDPI, Basel, Switzerland. This article is an open access article distributed under the terms and conditions of the Creative Commons Attribution (CC BY) license (https:// creativecommons.org/licenses/by/ $4.0 /)$.

\begin{abstract}
The adoption of pumps as turbines (PATs) in both small-scale hydroelectric plants and water supply systems has brought about various advantages, the most recognized being cost-effectiveness compared to other hydroturbines. However, due to their lack of flow control ability, their intolerance to off-design operations constitutes a tough shortfall. Moreover, since this technology is new, PAT flow dynamics are not yet well understood. Therefore, this study intends to numerically investigate the mixed-flow PAT's pump mode flow dynamics for five operating conditions from optimum $\left(1 \mathrm{Q}_{\mathrm{BEP}}\right)$ to deep part-load $\left(0.41 \mathrm{Q}_{\mathrm{BEP}}\right)$ conditions. Moreover, the effect of runner blade angle on them is investigated, considering three angles, namely $-2^{\circ}, 0^{\circ}$, and $2^{\circ}$. PAT flow stability was found to deteriorate as the flow decreased, where associated pressure pulsation level worsened at different flow zones. In addition, the blade angle increase led to correspondingly increasing flow unsteadiness and pressure pulsation levels, where the pulsation frequencies from rotor-stator interactions were dominant for most flow zones. This study's findings are of crucial importance to both scientific and engineering communities as they contribute to a thorough understanding of PAT flow dynamics.
\end{abstract}

Keywords: blade angle; flow unsteadiness; numerical simulation; pressure pulsation; pump as turbine

\section{Introduction}

The usage of hydraulic pumps in energy generation systems, which is generally known as the pump as turbine (PAT) technology, has brought about various advantages that improve the feasibility of energy supply projects at remote localities far from the electric grid reach [1-3]. In addition, this technology can be used to alleviate pressure levels within water distribution networks, at the same time producing usable electric power. This results in considerable energy savings [4-7]. As mentioned in different published works, PATs outperform similar microscale hydroturbines in terms of one main feature, namely cost-effectiveness [8-12]. Otherwise, PAT-associated technical shortfalls such as the intolerance of off-design operating conditions or the widely known lack of accurate conversion means between pumping and turbining performance characteristics would hinder its adoption [13-15]. In line with this, several studies have been carried out, addressing the already available techno-scientific issues within both of PAT's operating modes [15-17]. In this respect, in addition to PAT performance prediction studies [18-22], investigations into PAT flow unsteadiness under off-design operating conditions and respective flow structure formation mechanism have been carried out, which in the long run should lead to the improvement in terms of PAT's well-balanced performance in both of its operating modes. Among the recently published details on the same topic, a drop in 
PAT's mechanical efficiency has been recorded under part-load conditions, as a result of flow detachment and swirling flows that occurred within the machine flow channels [23]. In their study, $\mathrm{PAT}^{\prime}$ s BEP Flow rate $\left(\mathrm{Q}_{\mathrm{BEP}}\right)$ and Head $\left(\mathrm{H}_{\mathrm{BEP}}\right)$ under turbine mode have been found to overtake the pump mode by $27 \%$ and $41 \%$, respectively. In the same respect, Shen et al. [24], using the entropy generation theory for PAT's pump mode, showed the impeller and diffuser to be the main flow domains with higher mechanical energy losses, accounting for $35.32 \%$ to $55.51 \%$ and $32.61 \%$ to $20.42 \%$, respectively. These ones were found to be closely associated with the machine hump characteristics and became more and more pronounced as the machine flow rate decreased. It is also worth noting that the increase in tip clearance only worsened the turbulent dissipation at the impeller blade's tip area, leading to poorer machine performance. Both Ghorani et al. [25] and Lin et al. [26] used the above entropy theory to analyze PAT energy losses under turbine operating mode and dissect the most influential flow zones. Both studies have again converged to the realization that the runner and the volute casing flow zones constitute the flow zones of higher hydraulic losses, where for instance, Ghorani et al. [25] highlighted that $50 \%$ of energy dissipation could take place within the runner itself, owing to the complex flow phenomena that occurred, such as inter-blade backflow from the emerged inter-blade vortices and flow separations at blade leading and trailing edges, among others. Trying to improve the PAT performance under partial, full, and over-load conditions, Ntiri et al. [27], utilizing an optimization scheme, managed to improve the machine's efficiency by $23.7 \%$, $11.5 \%$, and $10.4 \%$ for the above-mentioned operating conditions, respectively. Subsequently, the inter-blade flow field for the optimized model attained a far better state, where under partial load for instance, runner inter-blade pressure distribution was uniform and vortices were considerably weakened. In another study [28], an attempt was made to optimize both the turbine and pump mode under design flow conditions for one rotational speed, and the obtained results were quite satisfying. After a realization that PAT back-curved blade design does not match the turbine mode operations where flow separations at the blade leading edge (BLE) are almost unavoidable, Wang et al. [29] designed a special runner with forward-curved blades for use under PAT's turbine mode, where the increase in blade inlet angle led to higher BEP flow conditions. Compared to the normal design, the new design exhibited a $13.2 \%$ improvement in terms of the machine's best efficiency. On the other hand, the clocking effect on PAT performance considering a diffusor kind of pump has been investigated by Hongyu et al. [30], while the effect of rotating speed was studied by Tahani et al. [31]. The same parameter was analyzed by Han et al. [32], but now studying the effect of the same on tip leakage vortex, considering a mixed-flow PAT in pump mode, where it was generally found that the increase in rotating speed worsens the tip leakage vortex.

Speaking of both axial and mixed flow PATs flow dynamics, which to the knowledge of the authors constitute the less-discussed types in this field; a number of results have been published where the focus is mostly put on tip leakage vortex dynamics [33,34]. In order to get a thorough understanding of PAT's turbine mode of operation and associated flow dynamics, especially considering a mixed flow PAT, one would first investigate its pump mode characteristics and the effect of different parameters on it. In this respect, the present article presents a numerical study that investigates the local flow structures formation mechanism and their eventual changes as the machine flow conditions change from part-load to full-load, primarily keeping the focus on PAT's pumping mode. In addition, an attempt was made to also investigate the effect mechanism of runner blade angle on both local flow dynamics and associated pressure pulsation characteristics, where three blade angles, namely $-2^{\circ}, 0^{\circ}$, and $2^{\circ}$ were considered. Unlike many of the recently published works about axial and mixed flow PATs, the present study considers a mixedflow PAT with no blade tip clearance. This article starts with an introductory section where details about the present study's research background are discussed and research motives are presented. Next, in the article's second section, the utilized PAT's physical model is described and the adopted numerical simulation scheme is explained and validated. In the 
third section, this study's results in terms of PAT flow dynamics and associated pressure pulsation characteristics are presented and discussed. Finally, concluding remarks are drawn and presented in the fourth and last section of the article. This study and its findings contribute to a deep understanding of PAT flow dynamics, starting with its pump mode.

\section{PAT Physical Model and Numerical Method}

\subsection{PAT Geometric Model}

In this study, the investigated PAT model is a mixed flow type with a high specific speed $(\mathrm{Nq}=163.9)$. It is composed of four main components, namely the inlet pipe, the runner, the diffuser, and the outlet pipe. The model's geometrical parameters are shown in Table 1 . The number of runner blades and guide vanes are 3 and 8 , respectively. For numerical simulations, the investigated computational domain, englobing the above-mentioned four components, has been built using a geometric modeling software, Unigraphix NX8.0. Figure 1 shows the utilized computational domain and components.

Table 1. PAT model geometric parameters.

\begin{tabular}{ccc}
\hline Parameter & Symbol & Value \\
\hline Runner Specific Speed (min $\left.{ }^{-1}\right)$ & $N_{Q}$ & 163.9 \\
Runner Inlet Dia. (mm) & $D_{\text {in }}$ & 300 \\
Runner Dia. (mm) & $D_{R}$ & 320 \\
Runner Blades Number (-) & $Z_{R}$ & 3 \\
Guide Vane outlet Dia. (mm) & $D_{O}$ & 350 \\
Guide Vanes number (-) & $Z_{G}$ & 7 \\
Blade tip clearance (mm) & $\delta$ & 0 \\
\hline
\end{tabular}

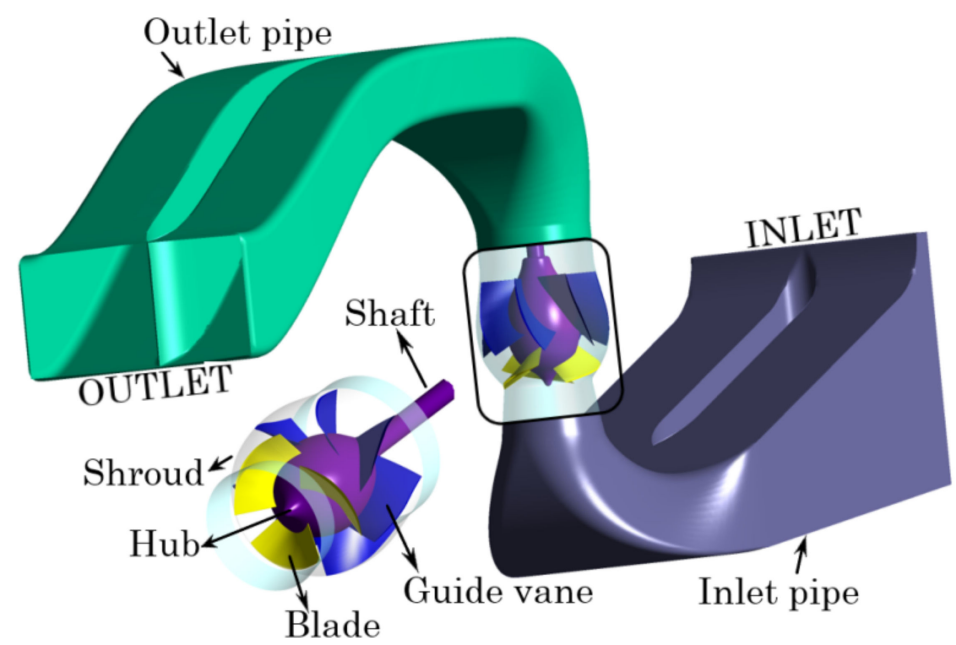

Figure 1. PAT computational domain and components.

Throughout the numerical simulations, the water is considered to enter the computational domain from the inlet zone of the inlet pipe, then successively passes through the runner and diffuser before exiting at the outlet pipe's outlet zone. This trajectory imitates the experimental process, where the flow followed the same trajectory. The investigated model, unlike many recently published works, presents no runner blade tip clearance. All the experiments have been conducted respecting the international standards for hydraulic machinery testing, i.e., the International Electrotechnical Commission standards or IEC [35]. Experimental tests on the machine's external performance characteristics were carried out for a wide range of flow conditions ranging from deep part-load, through part-load, upper part-load, and full load conditions, all the way up to over-load flow conditions.

Figure 2 shows the utilized experimental test rig and some components of the tested machine, while the schematic view of the testing system is given in Figure 3 . In Figure 4 , 
considering the runner blade angle of $-2^{\circ}$, experimental data in terms of head $(H)$, efficiency $(\eta)$, and shaft power $(P)$ are presented. The vertical line around the mid-zone of each graph is the BEP line. It can be seen that with a continuously increasing flow discharge, both the developed head and shaft power decreased, whereas the efficiency first increased until the BEP, before continuously dropping for further flow conditions. Having conducted experimental tests on a wide range of flows, the highest efficiency $(80.84 \%$.) was reached under $319.49 \mathrm{l} / \mathrm{s}$ flow conditions. This condition is characterized by the developed head of $8.52 \mathrm{~m}$ and a shaft power of $33.04 \mathrm{~kW}$.
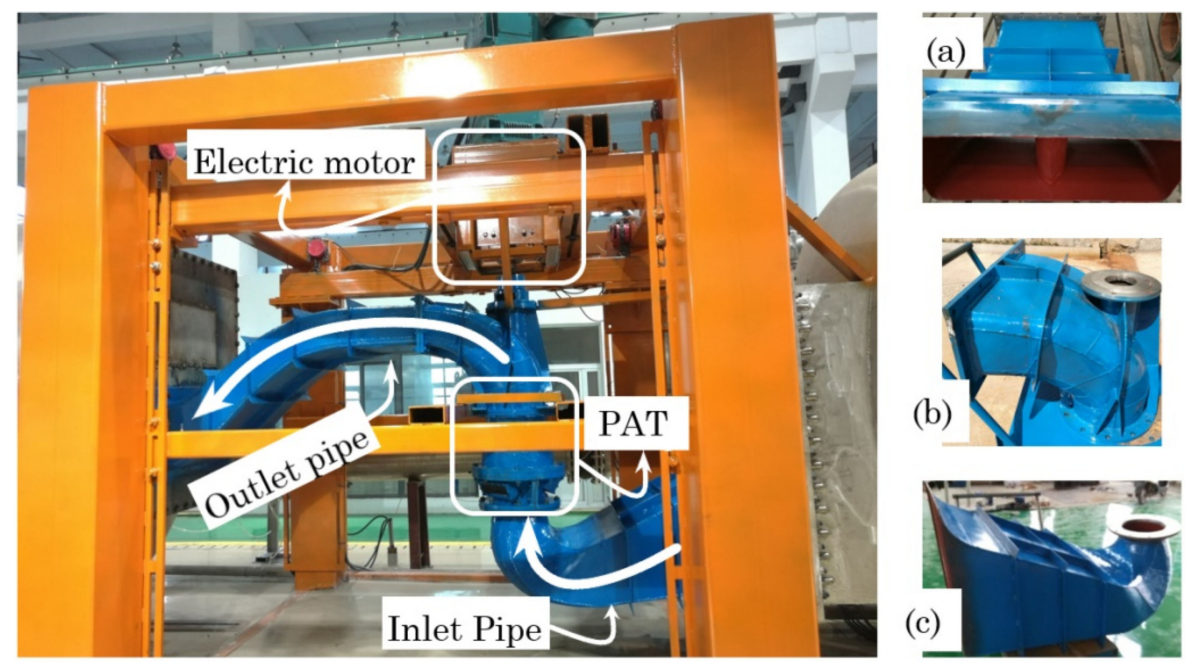

Figure 2. Experimental test rig and components (a) outlet pipe, (b) shaft zone, and (c) inlet pipe.

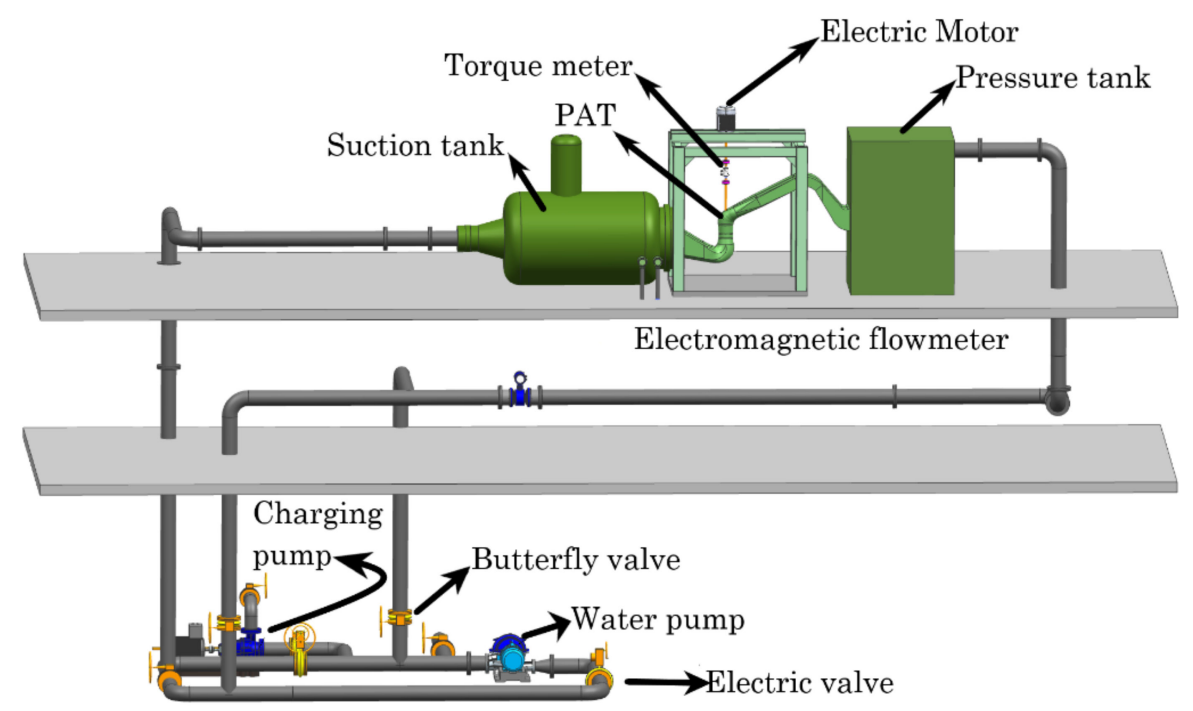

Figure 3. Schematic diagram of the PAT testing system. 


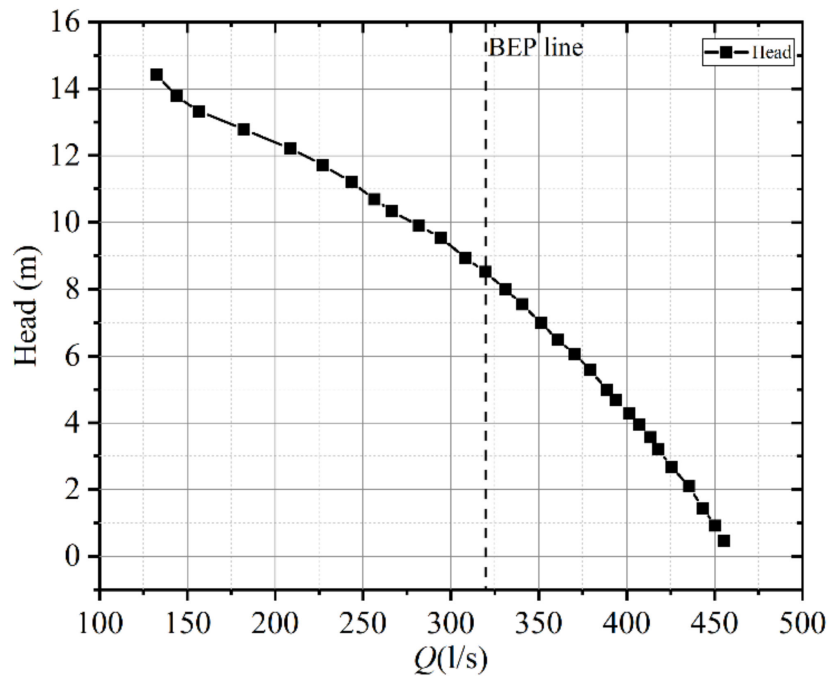

(a)

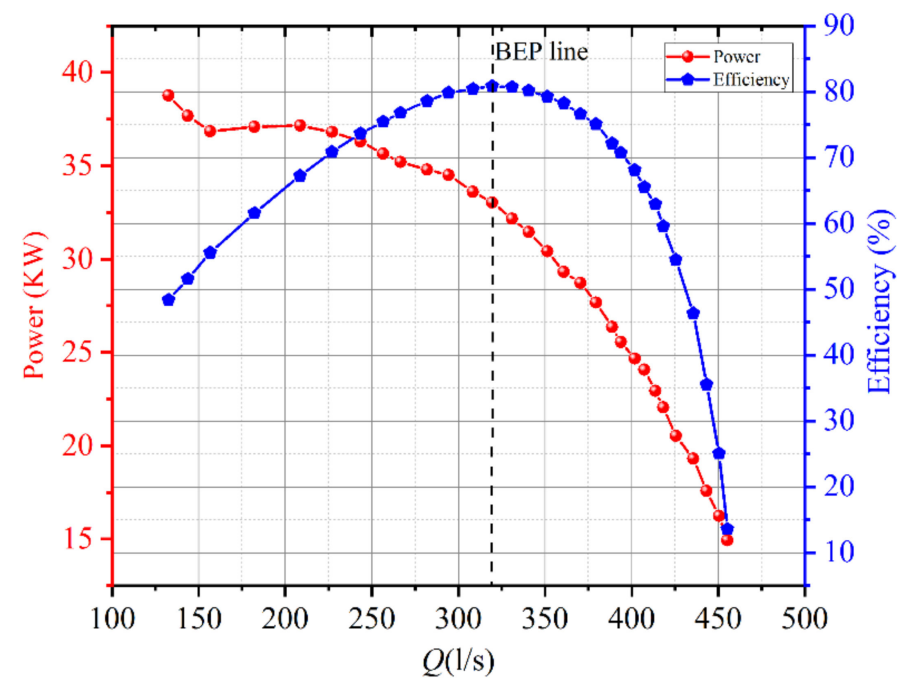

(b)

Figure 4. Machine external performance characteristics (a) $H$ - $Q$ curves (b) $\eta$ - $Q$ curves.

\subsection{Numerical Method and Validation}

\subsubsection{Computational Grid Generation}

In line with this study's main objectives, numerical simulations were conducted to investigate the machine flow dynamics and their evolution mechanism. As a preparatory phase, a computational grid has to be generated for the whole computational domain of concern. In this study, Ansys ICEM software was used to generate the computational grid for each of the four PAT components. Depending on components' geometric complexity, both tetrahedral and hexahedral schemes were utilized. A comparatively fine mesh was generated at flow zones of high importance such as the runner blades and guide vanes or any other flow deflection zones (turns or corners), leading to a global maximal y+ value less than 50 .

For the sake of eliminating the possibility of the utilized grid number influencing numerical simulation results, a grid independence test was been conducted where eight different grid numbers ranging from 5.4 to 9.7 million were tested. Steady-state numerical simulations were conducted on each grid set under similar boundary conditions, after which resultant performance characteristics in terms of head $(H)$ were compared. As shown in Figure 5, the developed head continuously increased with the grid number for all grid numbers below 8.92 million, while it almost stabilized for grid numbers above. Therefore, in line with the available computational resources, the grid number of 8.92 million was chosen for further simulations. Table 2 shows the details of the selected grid at different components of the investigated PAT model, while Figure 6 gives a quick glance on the generated mesh at all flow zones. 


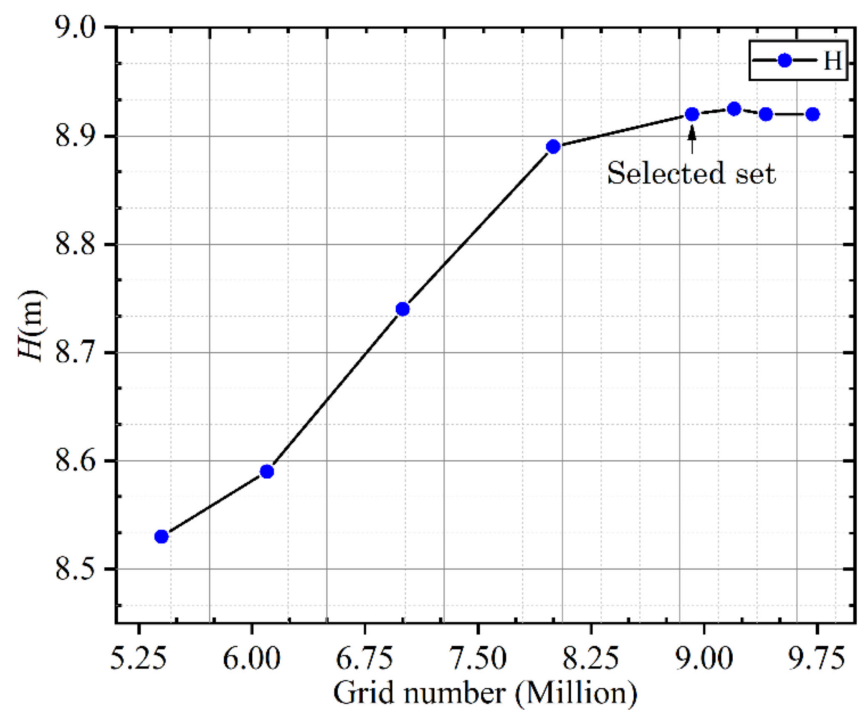

Figure 5. Grid independence test.

Table 2. Selected grid details for each PAT component.

\begin{tabular}{ccccc}
\hline Grid Details & Inlet Pipe & Runner & Guide Vanes & Outlet Pipe \\
\hline Grid type & Hexahedral & Tetrahedral & Tetrahedral & Hexahedral \\
Grid number & 1.2 & 3.41 & 3 & 1.31 \\
(Million) & & & & \\
Orthogonal & 0.45 & 0.3 & 0.4 & 0.4 \\
Quality (0-1) & 0.45 & 0.3 & 0.4 & 0.35 \\
Skewness $(0-1)$ & &
\end{tabular}
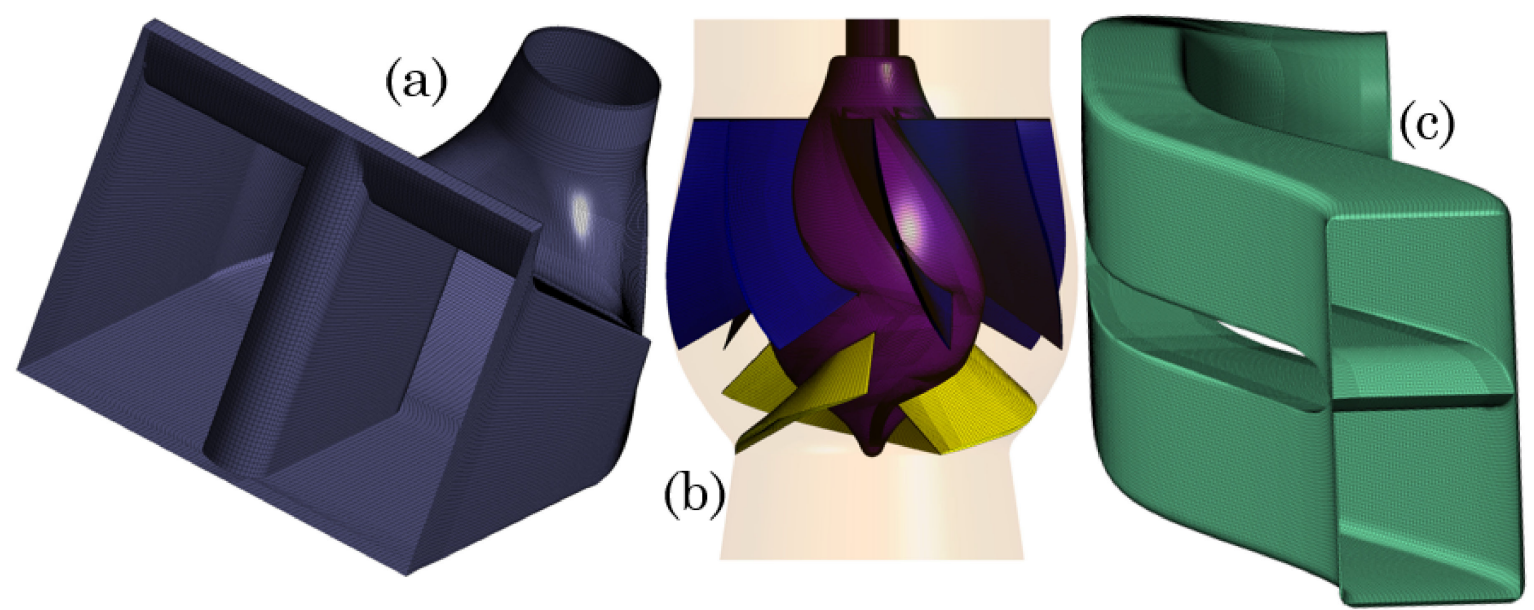

Figure 6. Selected grid for different model components (a) inlet pipe, (b) diffuser and runner, and (c) outlet pipe.

\subsubsection{Numerical Simulation Scheme and Validation}

The 3D numerical simulations on PAT flow under different operating conditions were carried out using the CFD commercial code Ansys CFX. This code, using the Shear Stress Transport turbulence model, solves the Reynolds-Averaged Navier-Stokes (RANS) equations, which guide the flow of incompressible as well as compressible fluids.

$$
\frac{\partial u_{i}}{\partial x_{i}}=0
$$




$$
\rho u_{j} \frac{\partial}{\partial x_{i}}\left(u_{i}\right)=\rho F_{i}-\frac{\partial p}{\partial x_{i}}+\mu \frac{\partial^{2} u_{i}}{\partial x_{j} \partial x_{j}}
$$

The investigated system, being a case of an axial flow PAT operating in pump mode, uses natural water at ambient temperature $\left(25^{\circ} \mathrm{C}\right)$, is classified as an incompressible fluid system. The continuity and RANS equations for incompressible flows are written in their Cartesian form in Equations (1) and (2), respectively. In these expressions, $\rho$, $\mu, p$, and $F_{i}$ are the fluid density, viscosity, pressure, and body forces, respectively. The SST $k$ - $\omega$ turbulence model, developed by Menter in 1993 [36,37], combines the advantages of two former and commonly used computational models, namely the $k-\varepsilon$ and $k-\omega$ models $[38,39]$. This combination has led to SST's ability to quite reasonably predict flow dynamics within the wall vicinities as well as the flow zones far from the wall. This model is also known for its good performance, especially when dealing with simulation cases with adverse pressure gradients and flow separations [40]. The associated expressions of turbulent kinetic energy, specific dissipation rate, and kinematic eddy viscosity are given in Equations (3)-(5), respectively.

$$
\begin{gathered}
\frac{\partial k}{\partial t}+u_{j} \frac{\partial k}{\partial x_{j}}=P_{k}-\beta^{*} k \omega+\frac{\partial}{\partial x_{j}}\left[\left(v+\sigma_{k} v_{T}\right) \frac{\partial k}{\partial x_{j}}\right] \\
\frac{\partial \omega}{\partial t}+u_{j} \frac{\partial \omega}{\partial x_{j}}=\alpha S^{2}-\beta \omega^{2}+\frac{\partial}{\partial x_{j}}\left[\left(v+\sigma_{\omega} v_{T}\right) \frac{\partial \omega}{\partial x_{j}}\right]+2\left(1-F_{1}\right) \sigma_{\omega, 2} \frac{1}{\omega} \frac{\partial k}{\partial x_{i}} \frac{\partial \omega}{\partial x_{i}} \\
v_{T}=\frac{a_{1} k}{\max \left(a_{1} \omega, S F_{2}\right)}
\end{gathered}
$$

During the 3D numerical simulation of PAT's flow under different conditions, targeting a quick and smooth solution convergence, steady-state numerical simulations were first conducted, the results of which served as the initial conditions for transient numerical simulations. For each simulation session, the values of utilized boundary conditions were picked from the above presented experimental data. Although experimental tests have been conducted on a wide range of flows $(Q)$ and blade angles $(\varphi)$, the present study only focuses on a small number of flow conditions while considering a single blade angle $\left(\varphi=-2^{\circ}\right)$. The investigated operating conditions range from part-load to full load (1 $\left.\mathrm{Q}_{\mathrm{BEP}}\right)$, where four cases have been considered for part-load operating zone, namely the deep part-load (0.41 $\left.\mathrm{Q}_{\mathrm{BEP}}\right)$, part-load (0.65 $\left.\mathrm{Q}_{\mathrm{BEP}}\right)$, and upper part-load (0.83 $\mathrm{Q}_{\mathrm{BEP}}$ and $0.92 \mathrm{Q}_{\mathrm{BEP}}$ ) conditions. However, in order to investigate the effect of blade design on PAT flow instability, the runner blade angle was increased for two successive values, namely $\varphi=0^{\circ}$ and $\varphi=+2^{\circ}$, considering the most unstable flow conditions. Throughout the simulation process, the whole PAT computational domain was divided into stationary frame (inlet pipe, outlet pipe, and diffuser) and rotating frame (runner), between which different interfaces were used. For instance, the frozen rotor-stator interface was utilized between the runner and the diffuser and between the runner and the inlet pipe for steadystate numerical simulations. For transient numerical simulation, the frozen rotor-stator interface was replaced by the transient rotor-stator interface at the same flow zones. For other interfaces linking stationary components such as the interface between the diffuser and outlet pipe, the general grid interface (GGI) was used. In addition to $P_{\text {in }}$ and $Q_{\text {out }}$ as inlet and outlet boundary conditions, the non-slip wall condition was imposed at all other walls of the concerned computational domain. A high-resolution scheme was chosen for both the advection scheme and turbulence numerics. For each transient solution, the timestep $\Delta t$ has been set as the time required for the runner to rotate 1 degree, which means that one full runner rotation is equivalent to $3600 \Delta t$. Moreover, five internal loops were selected for each timestep. Every solution was set to last for ten runner rotations, where the solution's convergence criterion was set as the residuals Root-Mean Square below $10^{-5}$. For the sake of checking the validity of numerical results obtained using the above presented numerical simulation scheme, simulation results in terms of the machine's 
external performance characteristics, viz. developed head $(H)$, efficiency $(\eta)$, and power $(P)$, were compared to experimental ones as shown in Figure 7 . To do so, numerically found variations at each of the above three parameters were averaged to come up with final parametric values. Under normal operating conditions, the error between numerical and experimental data is globally found to be around $5 \%, 1 \%$, and $2 \%$ for head, efficiency, and power predictions, respectively. However, due to serious flow separations and associated adverse pressure gradients that take place under off-design operating conditions, there is an increase in computational errors for flow conditions away from the BEP. Nevertheless, on a global scale, numerical results obtained using this numerical scheme can reasonably compare to a real-life situation. Note that the formulas utilized for head, power, and efficiency are shown in Equation (6), where $P_{2}, P_{1}, \rho, g, n, T$, and $Q$ stand for outlet pressure $(\mathrm{Pa})$, inlet pressure $(\mathrm{Pa})$, gravitational acceleration $\left(\mathrm{m} / \mathrm{s}^{2}\right)$, runner rotational speed $(\mathrm{rpm})$, torque $(\mathrm{Nm})$, and flow discharge $\left(\mathrm{m}^{3} / \mathrm{s}\right)$, respectively.

$$
H=\frac{P_{2}-P_{1}}{\rho g} P=\frac{2 \pi n T}{60} \eta=\frac{\rho g Q H}{P} \times 100
$$

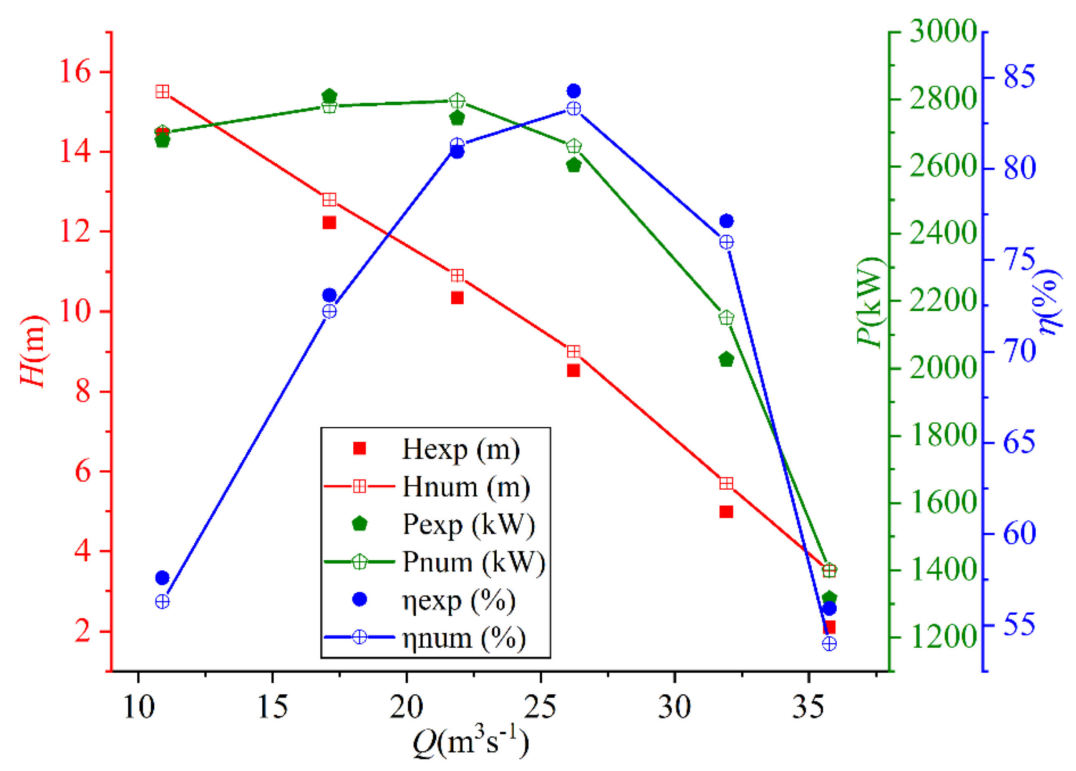

Figure 7. Comparison between numerical and experimental results.

\section{Results and Discussion}

\subsection{Flow Field Characteristics}

In order to investigate the eventual changes in terms of water flow dynamics with the runner for different flow conditions, five turbo-planes within the runner inter-blade flow channels have been considered, from the hub side towards the shroud side as SP1, SP2, SP3, SP4, and SP5. Considering the unitary spanwise distance from the runner hub to the shroud, these planes occupy five successive positions as $0.1,0.3,0.5,0.7$, and 0.9 , respectively. In Figure 8 , contours of runner flow velocity distribution and corresponding vorticity measure under different flow conditions have been presented. Gradually increasing the machine influx from deep part-load flow conditions to the BEP, the highest flow speed was recorded at flow zones within the vicinities of the runner shroud, while the lowest was situated close to the hub. Moreover, velocity contours show that higher-velocity flows on individual turbo-planes are recorded both on the runner blade's suction side and the runner outlet zones (ROZ). It is also obvious that high-speed flow zones on the blade suction side (BSS) become larger along the spanwise direction towards the shroud, and extends farther in the streamwise direction towards downstream flow zones. 


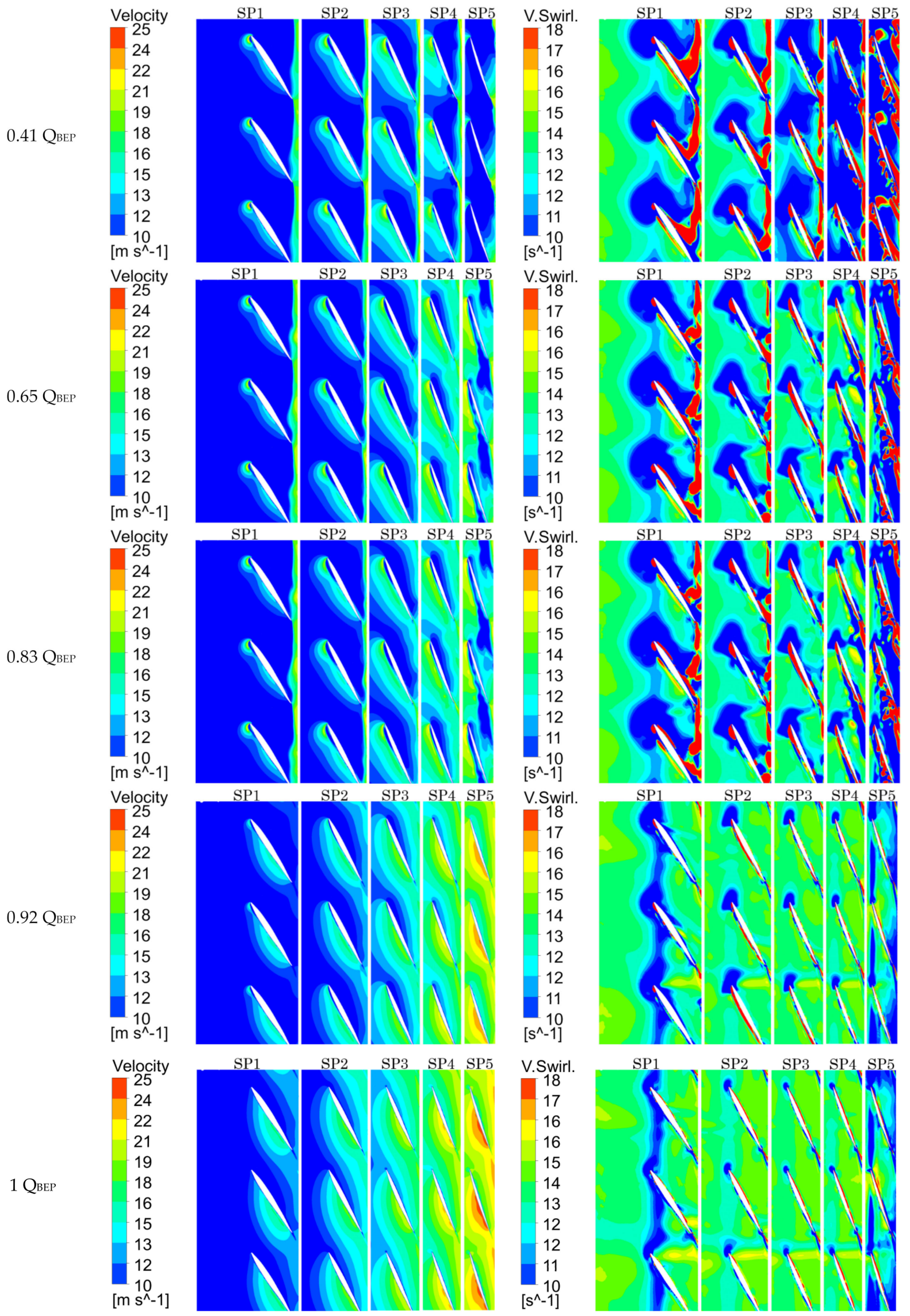

Figure 8. Contours of flow velocity and vorticity within the runner inter-blade flow zone for different flow conditions. 
Moreover, the increase in machine influx led to a continuous enlargement of flow zones occupied by higher velocity flows on the blade suction side, where they reach the blade trailing edge (BTE) and start to expand towards inter-blade channels' mid-zone, finally touching the blade pressure side's (BPS) trailing zone under upper part-load and BEP flow conditions. This feature is believed to take the source from the flow separation that took place both at the runner blade's leading edge and the blade suction side vicinities in the downstream flow field. For deep part-load flow conditions, flow separation at blade suction side in downstream zones is amplified by inlet swirling flow, leading to its obvious detachment from the blade leading edge one in the vicinities of the runner hub. This detachment was, however, found to weaken in the spanwise direction, leading to a reattachment of both flow separation zones to form one huge zone expanding the whole blade length at flow zones in the vicinities of the runner shroud. Flow separation at the blade leading edge is also found to be linked with a large flow incidence angle under deep part-load conditions. It was found that, with the decrease in the machine flow discharge, the flow incidence angle at the runner blade's leading edge gradually increased, eventually resulting in the formation of huge flow wakes at the same zone, especially within both the runner blade's hub and shroud vicinities under low flow conditions.

Correspondingly, the representation of flow vorticity measure within the runner inter-blade zone using the velocity swirling strength parameter not only confirmed the aspects discussed above, but also highlighted the presence of two more high-vorticity zones, one attached to the runner blade's pressure side at the runner mid-length zone, the other at the blade trailing edge. The latter extends opposite to runner rotational direction, swiping the blade trailing edge zone in the form of wake structure and obviously occupying the above-mentioned high-speed flow zone within the runner outlet vicinities, which understandably makes connections with the vaneless space vortex flow, before meeting the next blade's pressure side vortex. It was also found that this high-vorticity zone at the runner outlet gradually narrows down in the spanwise direction until the mid-span zone, where it enlarges again towards the shroud vicinities. At this zone, blade trailing edge-attached wake-like vortices no longer extend far enough to reach the next blade's pressure side-attached vortex. Instead, the screw-like vortex emerges at the runner suction side, extending from the blade leading edge's shroud tip region, all the way to the downstream zone, where it gradually enlarges towards the runner outlet zone. This structure is attached to the runner shroud, and as any other discussed secondary flows, decays with the increase in machine flow discharge.

To shed more light on the aspects discussed above, in Figure 9, a visual is provided on the local pressure distribution mode on the runner blade's suction and pressure surfaces. Starting at the blade suction surface, local pressure distribution mode can be distinctly classified into two zones, namely the low-pressure zone at the runner blade's leadingedge and vicinities and the high-pressure zone at the blade's trailing edge and vicinities. However, there is a smooth transition from one zone to another from the blade leading edge towards the downstream, and each zone's size varies depending on the considered operating conditions. In good agreement with the velocity distribution mode discussed above, the low-pressure zone at the blade leading edge spans the whole spanwise blade length from hub to shroud and extends downstream to some blade mid-length distance. In addition, this zone extends farther in the shroud region than the hub region. This agrees well with the flow velocity distribution mode on the blade suction side, as discussed in the above sections, where it was shown that high-velocity zones are narrow in the hub zone but grow towards the downstream in layers above, such as the flow zones close to or within the shroud vicinities. These low-pressure zones close to the shroud are also found to grow further in the downstream direction with the increase in the machine flow discharge. The detachment discussed above of the high-velocity zones on the blade suction side from the influence of the inlet swirling flow in the hub zone is even obvious in Figure 9, where a correspondent high-pressure sector within the hub vicinities is shown to grow in size with 
the machine flow discharge. On the other hand, the situation on the blade pressure surface is the opposite to that of the suction surface.
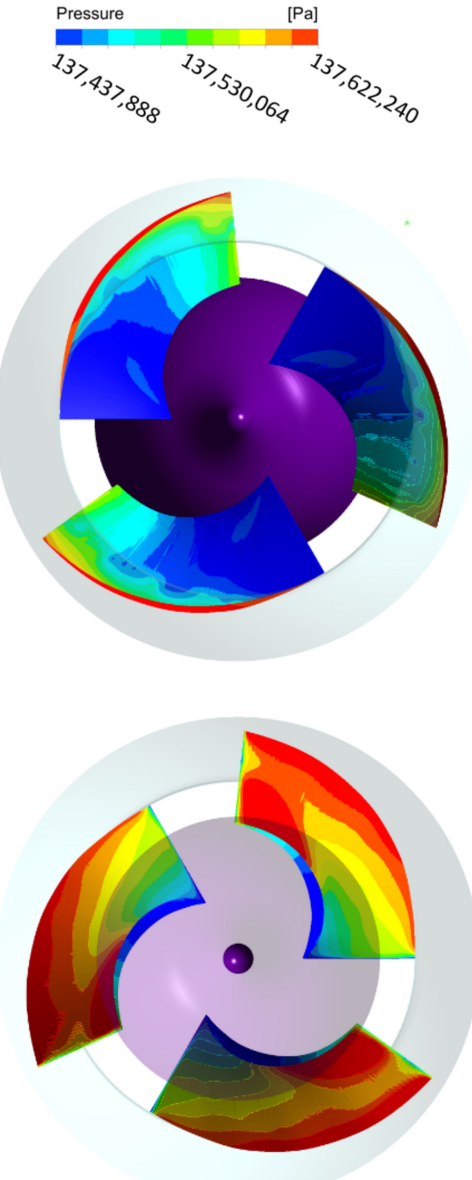

(a)
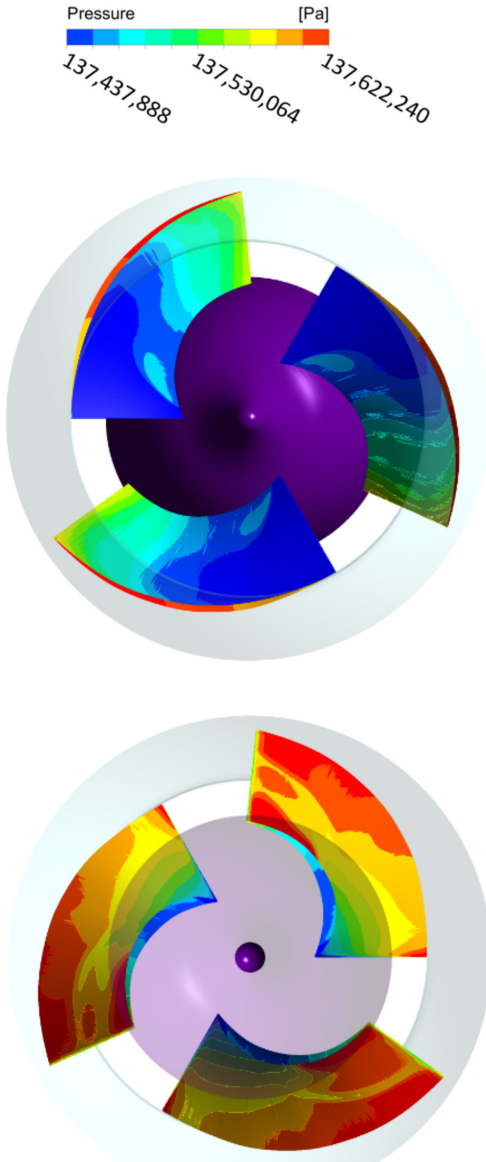

(b)
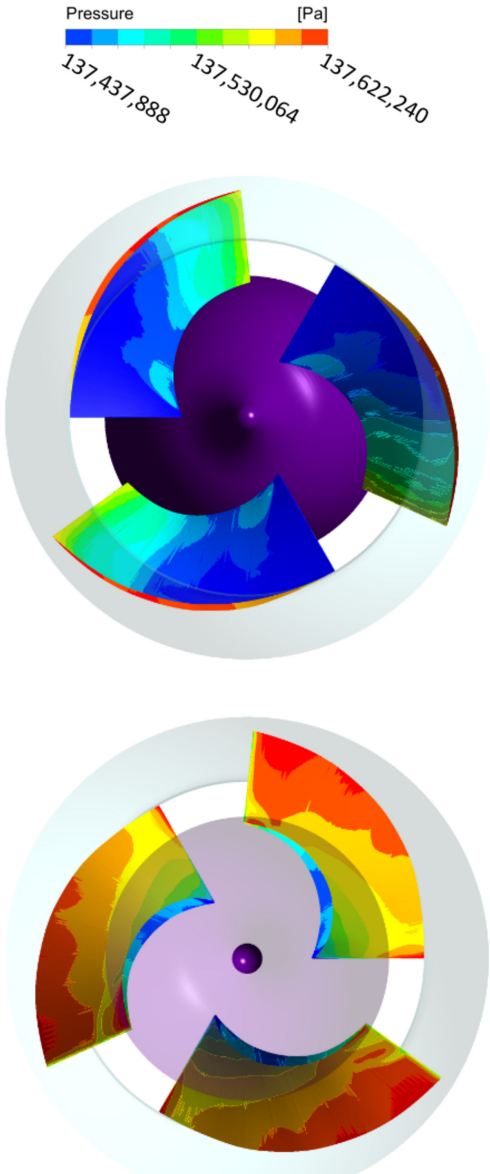

(c)

Figure 9. Contours of static pressure on the runner blade's suction (up) and pressure (down) sides under different flow conditions (a) $0.41 \mathrm{Q}_{\mathrm{BEP}}$, (b) $0.65 \mathrm{Q}_{\mathrm{BEP}}$, (c) $0.83 \mathrm{Q}_{\mathrm{BEP}}$.

On this side, the high-pressure zone is localized at the blade trailing edge vicinal zone and grows bigger from the hub towards the shroud zone. Moreover, the extent of the high-pressure zone in the shroud vicinities is found to decrease with the increase in machine flow discharge. This is in good agreement with the flow vorticity distribution mode discussed above and the associated evolution at the same zones.

In Figure 10, flow streamlines, colored according to velocity, on a transversal plane from the runner inlet to the outlet are presented for three successive instants, namely $t_{0}$, $t_{0}+T / 12$, and $t_{0}+T / 6$ respectively, where $t_{0}$ stands for the initial time and $T$ the runner rotational period. Considering three part-load operating conditions, this figure shows the instant changes that occurred in runner flow structures. It is globally shown that runner flow vorticity becomes larger and tougher with the decrease in machine flow discharge. Moreover, main flow vortical structures positions seem to remain unchanged despite the change in machine flow conditions. They rather grow or decrease in size. In this figure, one would notice both the BPS and BSS-attached vortices and their extensions in shroud vicinities away from the blade, as well as the runner outlet vortex, which is mostly within the vicinal zones of the runner hub. The latter is found to grow larger and expand to mid-span zone and beyond for deep part-load conditions. 


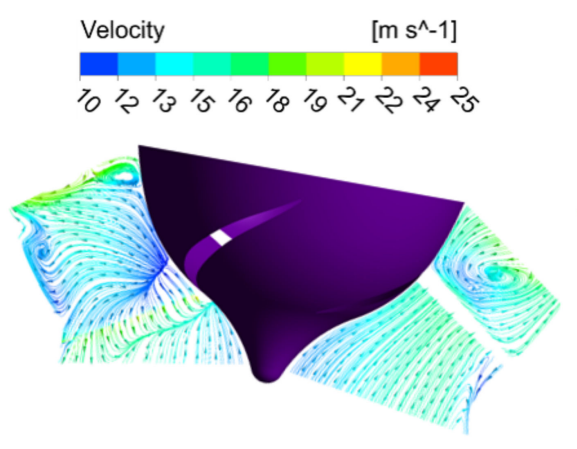

(a) 0.41 Qвер $\left(t_{0}\right)$

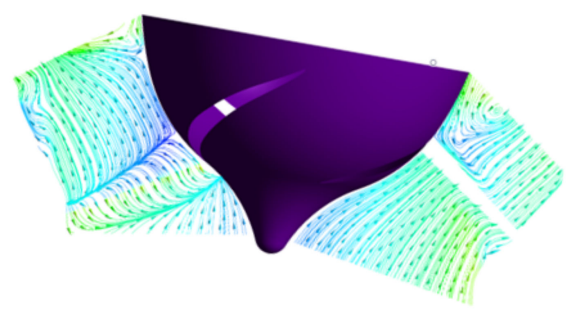

(a) 0.65 Qвер $\left(t_{0}\right)$

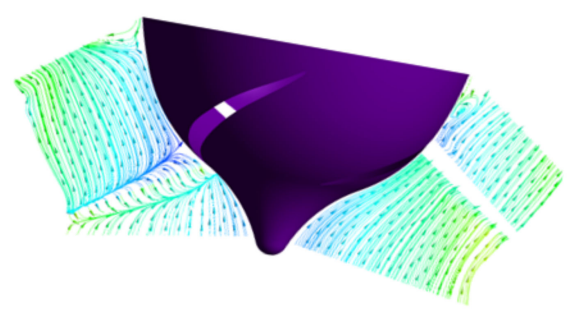

(a) $0.83 \mathrm{QBep}\left(t_{0}\right)$

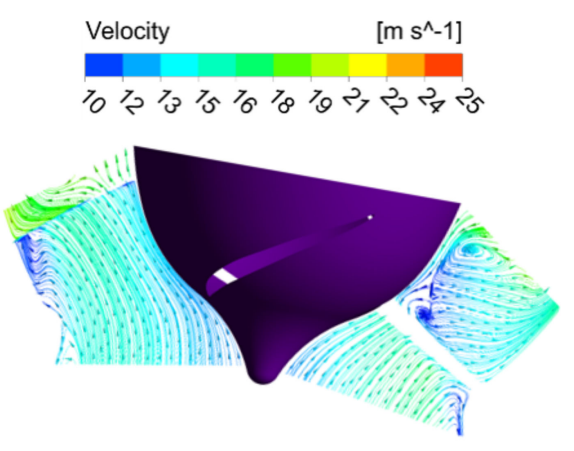

(b) $0.41 \mathrm{QBEP}\left(t_{0}+T / 12\right)$

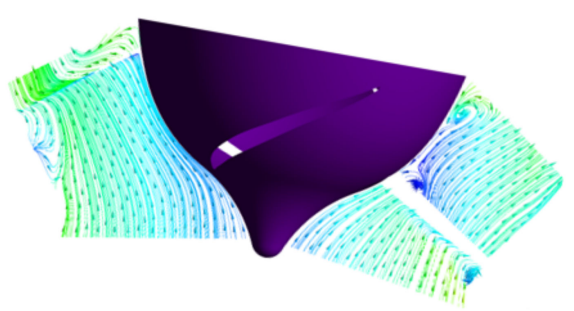

(b) 0.65 QвеР $\left(t_{0}+T / 12\right)$

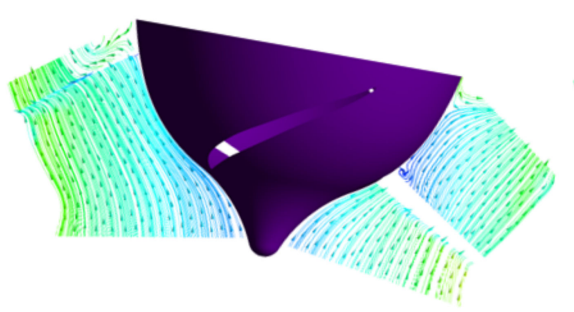

(b) $0.83 \mathrm{Qвер}\left(t_{0}+T / 12\right)$

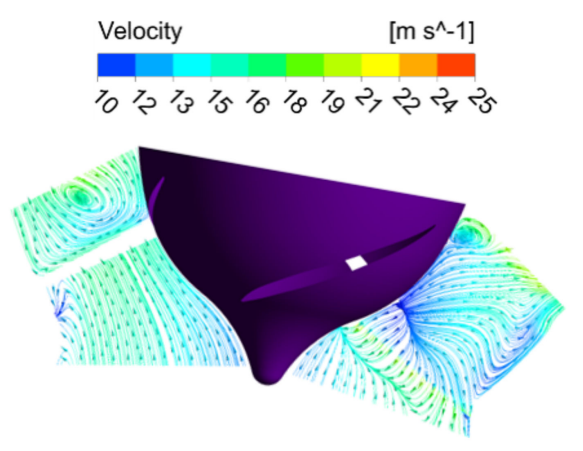

(c) 0.41 QвеP $\left(t_{0}+T / 6\right)$

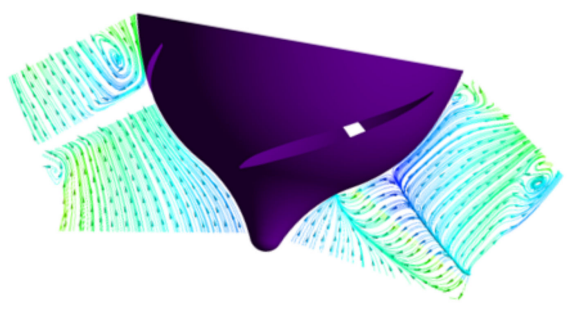

(c) 0.65 Qвер $\left(t_{0}+T / 6\right)$

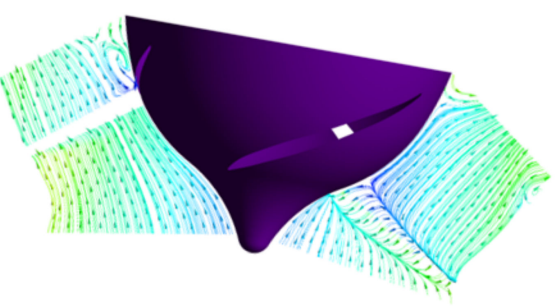

(c) $0.83 \mathrm{QBep}\left(t_{0}+T / 6\right)$

Figure 10. Streamlines colored according to velocity within the runner for different flow conditions at different instants.

Globally speaking, and in line with details as presented in Figure 11, the runner flow vorticity can be classified into four groups respective to their positions, namely the BLE vortex, BSS vortex, BPS vortex, and ROZ vortex. Figure 11 gives a view of the main vortex flow locations in the runner flow zone for the three investigated part-load conditions. Therefore, in this figure, the above-mentioned positions are marked by letters A, B, C, and $\mathrm{D}$, respectively. While the left part of the figure presents the runner assembly with main flow vortex positions indicated, the middle and right sides of the figure show the runner BSS and BPS with the corresponding flow vortices. In total agreement with the above sections, the BLE vortex (A) grows bigger in the spanwise direction, finally reaching the runner shroud region, from where the BSS vortex (B) starts and extends the whole blade length and beyond, but mainly staying within the shroud vicinal zone. Depending on the considered machine flow conditions, this structure may link up with the ROZ vortex (D) at the runner outlet and extend towards the opposite direction of the runner rotation to eventually connect with the preceding blade's BPS vortex (C). This is, for instance, the case for deep part-load condition as presented in Figure 11a. While all these vortices are found to weaken and eventually vanish as the machine flow conditions move from part-load to the rated operating conditions, the BPS vortex does the same while changing locations with different flow conditions. Under deep part-load conditions $\left(0.41 \mathrm{Q}_{\mathrm{BEP}}\right)$, the BPS vortex extends from blade mid-length to downstream. As the flow increases to part-load conditions (0.65 $\left.\mathrm{Q}_{\mathrm{BEP}}\right)$, it moves spanwise downward to mid-span zone and now extends from BLE to the runner outlet in a string-like form. 

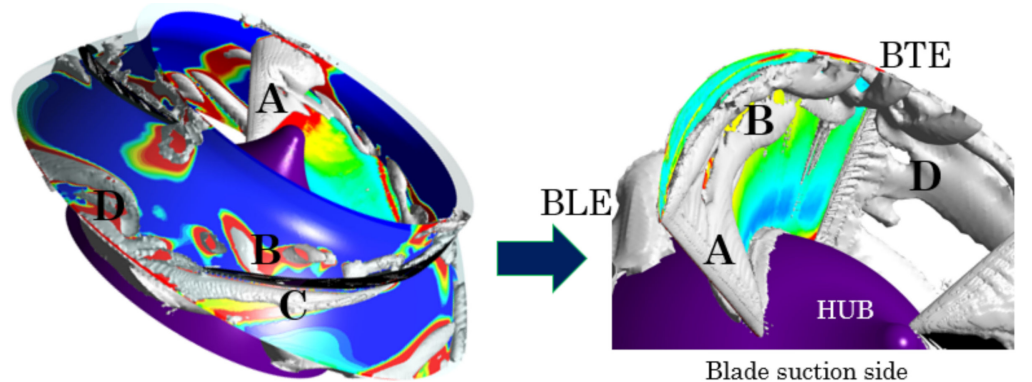

Blade suction side

(a) 0.41 QBEP
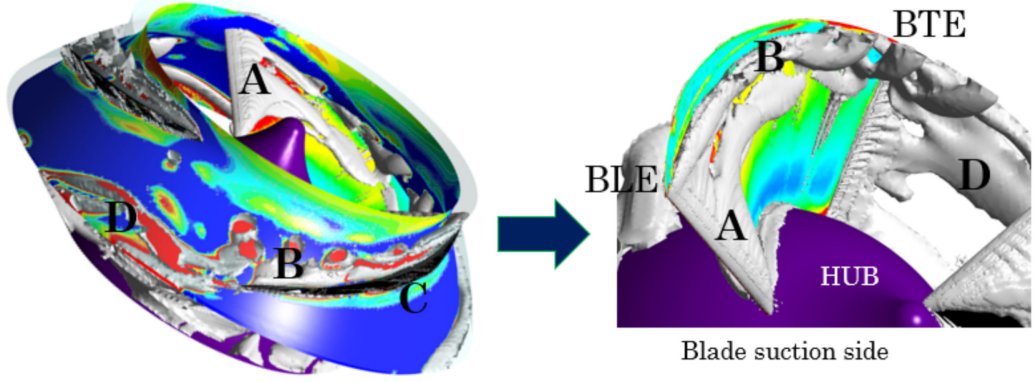

Blade suction side

(b) $0.65 \mathrm{QBEP}$

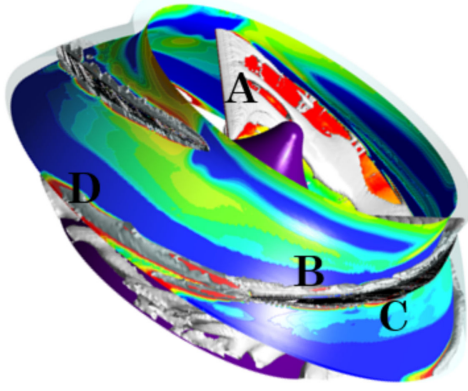

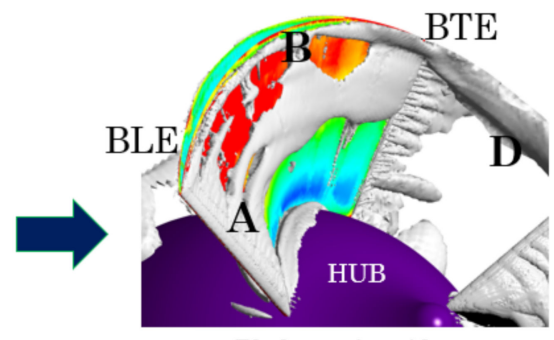

Blade suction side

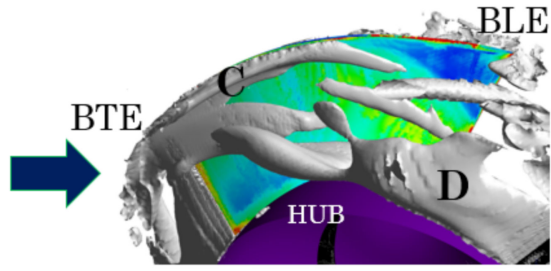

Blade pressure side

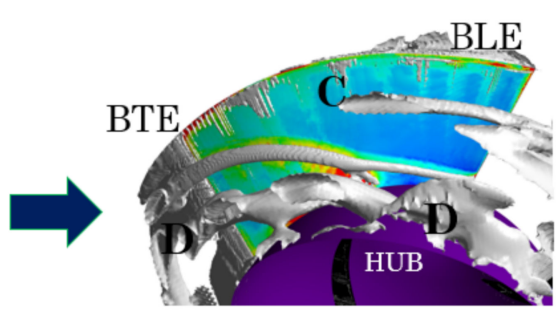

Blade pressure side

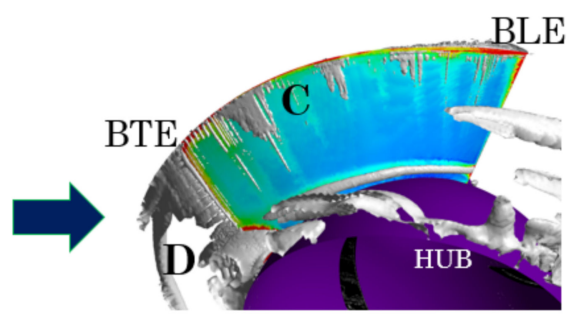

Blade pressure side

(c) 0.83 Qвег

Figure 11. Main flow vorticity zones within the runner for different flow conditions.

Under upper part-load conditions, it again moves downwards to the runner hub's vicinal zone and maintains a string-like form extending from the BLE to the ROZ. For the sake of analyzing the flow dynamics within inter-guide vane (GV) flow channels, the next four figures (Figures 12-14) give a visual of both velocity contours and corresponding velocity-colored streamlines within the diffusor flow zone for different operating conditions. To do so, three consecutive planes, SG1, SG2, and SG3, were selected in the streamwise direction at positions $1.15,1.55$, and 1.95, considering positions at the diffuser inlet and the diffuser outlet as 1 and 2, respectively. Under 0.41 Q $\mathrm{BEP}$ operating conditions, SG1, being the closest to the vaneless space between the runner and diffuser, exhibits a huge zone of very low flow velocity, expanding from the hub to beyond the mid-span zone and a narrow high-speed flow right at the diffuser's shroud zone. 

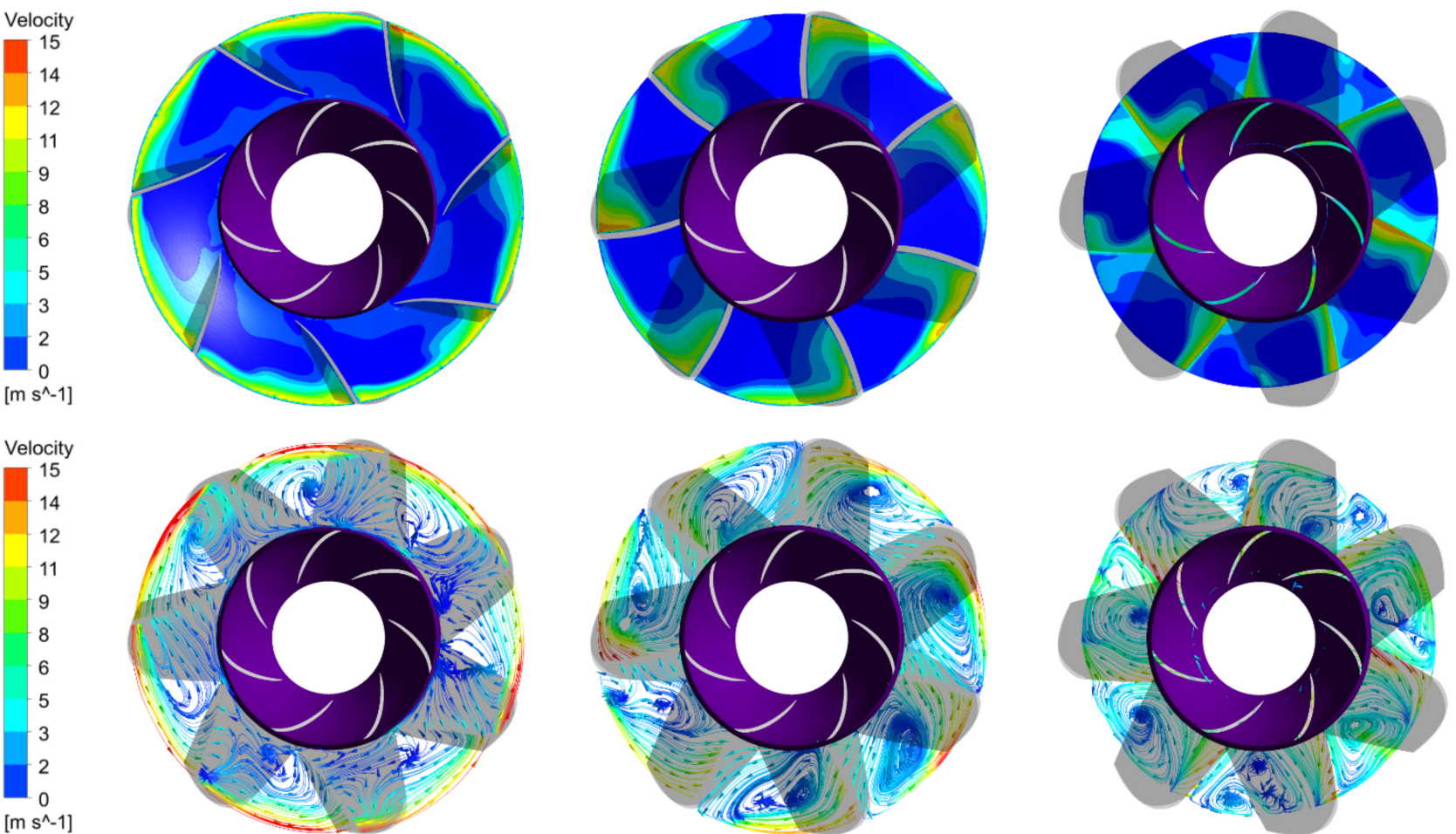

(a) SG1

(b) SG2

(c) (SG3)

Figure 12. Flow axial velocity contours and streamlines within the diffuser under $0.41 \mathrm{Q}_{\mathrm{BEP}}$ flow conditions for different positions along the streamwise direction.
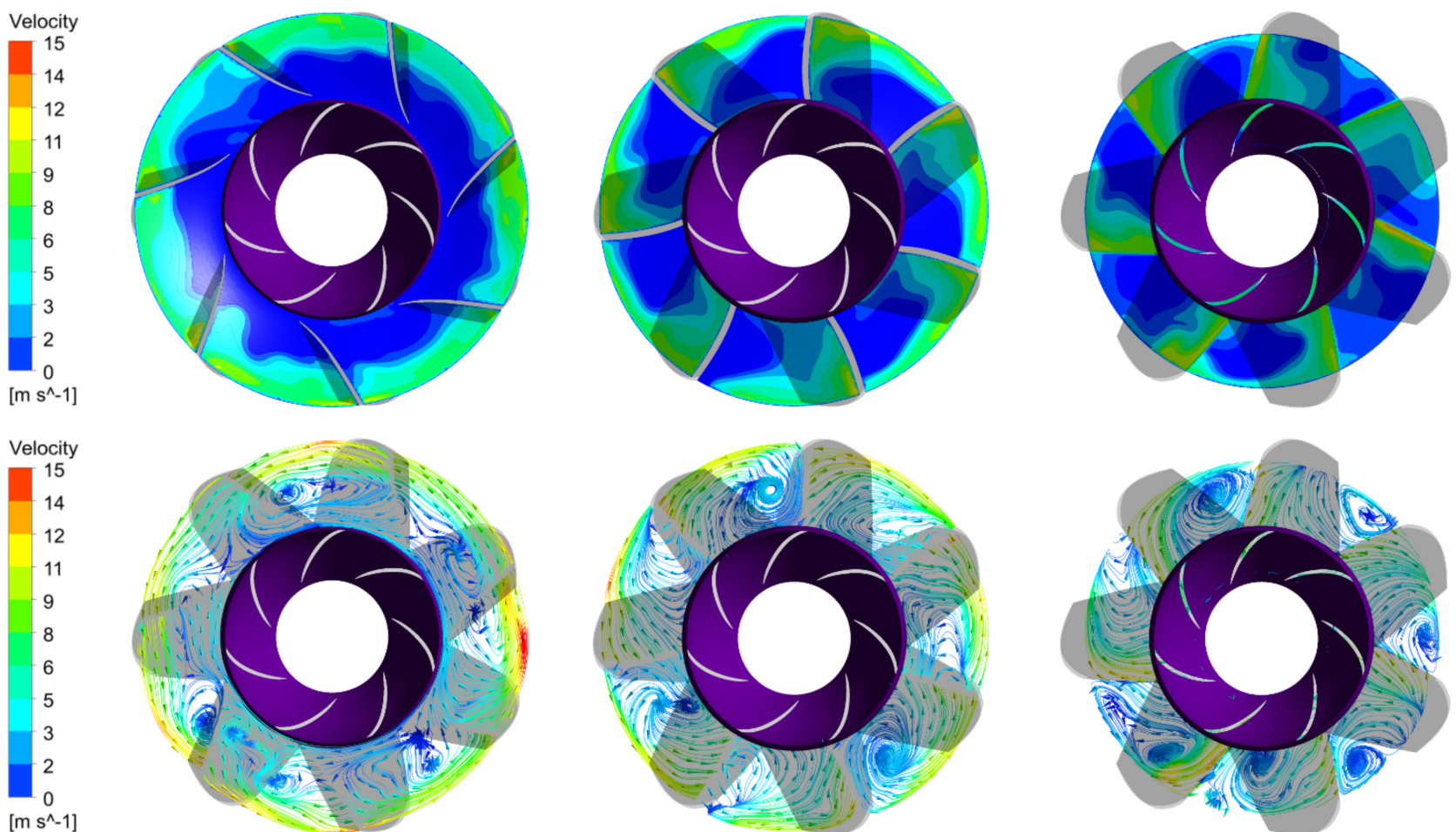

(a) SG1

(b) SG2

(c) (SG3)

Figure 13. Flow axial velocity contours and streamlines within the diffuser under $0.65 \mathrm{Q}_{\mathrm{BEP}}$ flow conditions for different positions along the streamwise direction. 


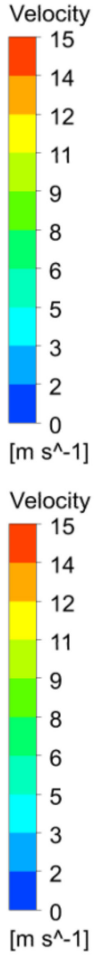

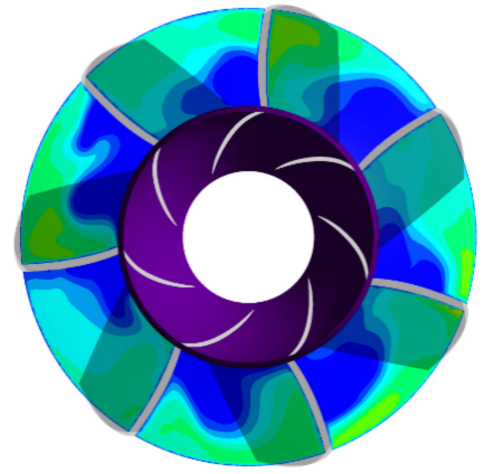
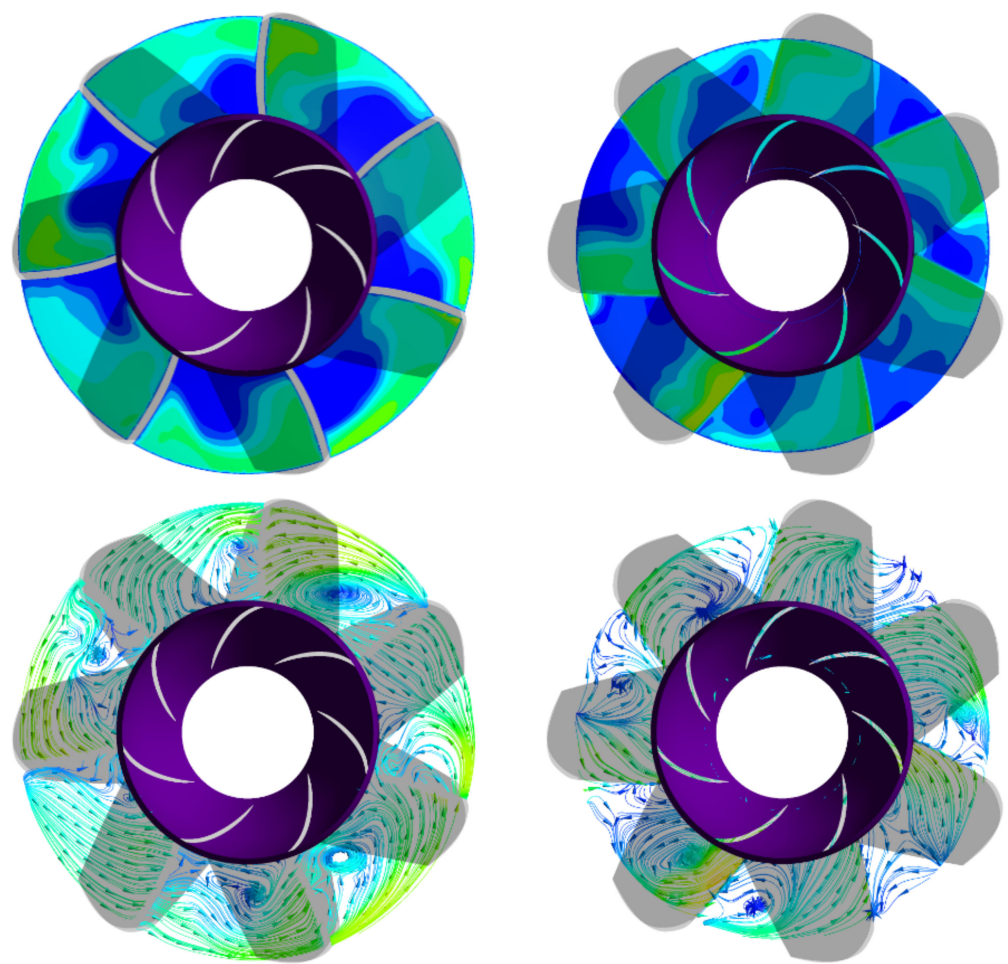

(b) SG2

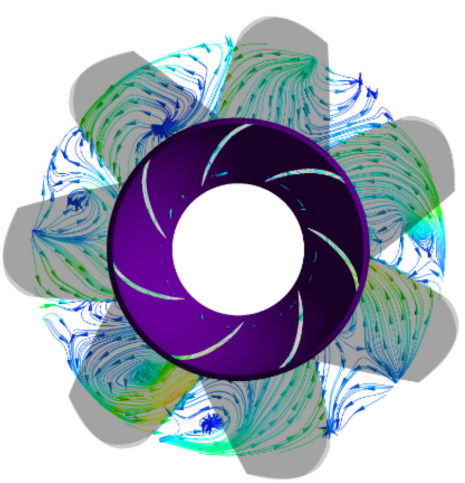

(c) SG3

Figure 14. Flow axial velocity contours and streamlines within the diffuser under 0.83 QBEP flow conditions for different positions along the streamwise direction.

As shown by correspondent velocity-colored flow streamlines, low flow-speed zones are marked by large flow vortices and subsequent backflows. At the diffuser inlet, a large vortex and backflow is distributed in all channels circumferentially and blocks all hub flow zones, thus pushing the water to tiny left outflow areas at the shroud, resulting in highspeed flows at the shroud and vicinal flow zones. At the diffuser mid-zone, flow vortices occupy a bigger portion from the central flow area to one bounding blade's pressure side, thus leaving the water flowing area at another blade's suction side and a small portion in the shroud vicinal zone. At the outlet, the emergence of a double vortex leads to the occupation of the channel's central zone, leaving tiny flowing areas attached to both bounding vanes' surfaces. As shown in the rest of the figures (Figures 13-15), the flow blockage by the emerged flow vortices and backflows close to the diffuser hub and vicinal flow zones at the diffuser inlet were weakened with the increase in machine flow discharge. Correspondingly, flow vorticity at other flow zones within the diffuser was gradually weakened, leading to almost no flow blockage at the diffuser inlet under rated conditions (see Figure 15).

At this zone, the high-speed flow zone is almost circumferentially distributed, where, however, in reality, a wider high-speed flow zone is attached on the guide vane's suction surface (GSS), extending towards the opposite direction to the runner rotation, eventually reaching the precedent guide vane's pressure surface (GPS) on its shroud tip region. At this zone, low-speed flow occupies almost $80 \%$ of the whole flow area. Under the same operating conditions, at the diffuser mid-distance in the streamwise direction (SG2 plane), a high-speed flow zone on the GSS detaches from the precedent GV and expands spanwise downwards, pushing the low-speed flow zone towards the preceding GV's pressure side. At this zone, low-speed flow occupies around $75 \%$ of the whole flow area. At the diffuser outlet (SG3 plane), the low-speed zone moves to the center of the channel, leaving tiny high-speed zones attached to both vanes' pressure and suction surfaces. At this zone, the low-speed flow zone occupies more than $80 \%$ of the available flow area. 

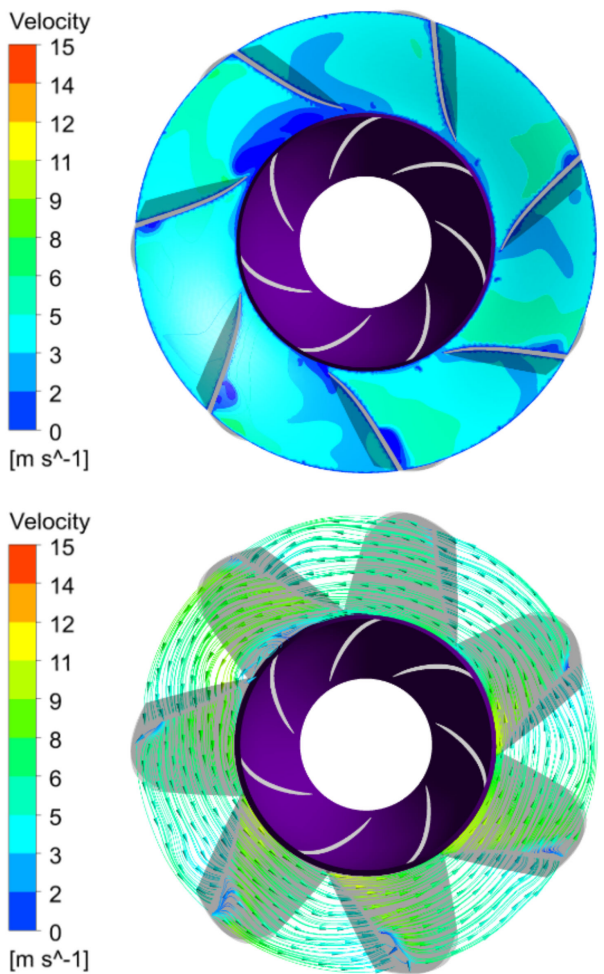

(a) SG1
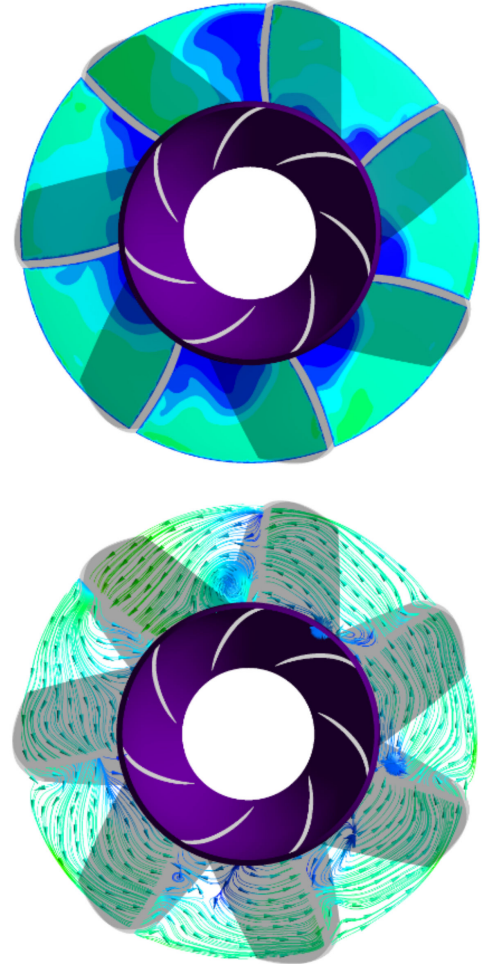

(b) SG2
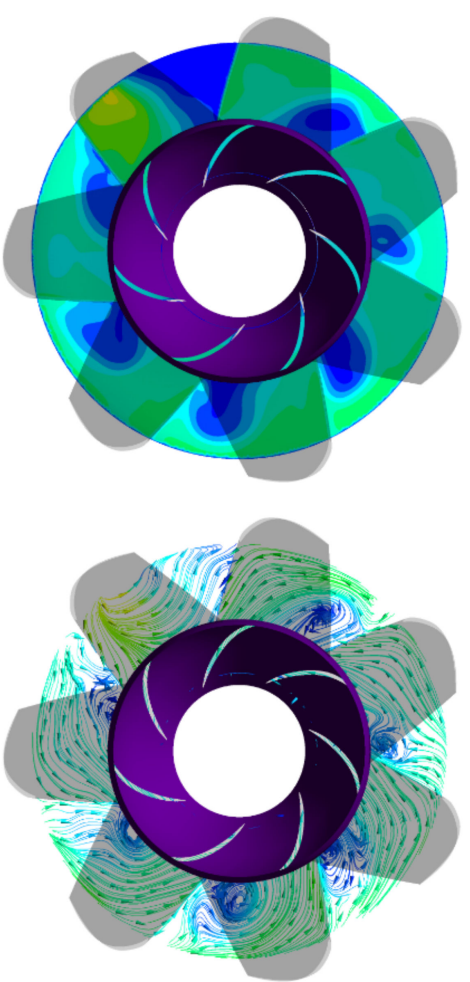

(c) SG3

Figure 15. Flow axial velocity contours and streamlines within the diffuser under 0.92 QBEP flow conditions for different positions along the streamwise direction.

\subsection{Pressure Field Characteristics}

In order to investigate the eventual pressure pulsation characteristics as associated with the flow dynamics discussed above, different pressure monitors were positioned at various locations along the PAT flow full flow passage. Figure 16 gives a visual of the placement of the mentioned pressure monitoring points within the runner flow zone. The specifically investigated zones are the inter-blade flow zones (IB), the runner blade's suction and pressure sides (BS and BP), and the vaneless space (VS) between the runner blades and the distributor's guide vanes. In more detail, nine monitors within the IB zone were positioned at three successive locations in the streamwise direction on the runner's mid-span plane, as shown in Figure 16a. Moreover, three spanwise layers were considered when positioning pressure monitors on the blade's both surfaces. These layers were located at positions $0.1,0.5$, and 0.9 , considering the positions of runner hub and shroud as 0 and 1 , respectively, where six monitors were considered on each layer, making it 36 monitors on both the BSS and BPS, as shown in Figure 16c. Finally, 24 monitors were equidistantly positioned on the mid-span plane within the vaneless space (Figure 16b).

Frequency domain pressure pulsation spectra within the vaneless space for the five investigated operating conditions are shown in Figure 17. However, for clarity, and owing to the fact that pressure pulsation characteristics within a specific flow zone under specific operating conditions are more likely to present little to no differences, only the first five VS monitors are presented. The vaneless space pressure pulsation characteristics are found to considerably vary with the changing machine operating conditions, where, for instance, the pressure pulsation amplitude of the dominant frequencies are continuously increased as the machine flow conditions decreased from rated conditions to part-load, before dropping to almost a half under deep part-load conditions. 


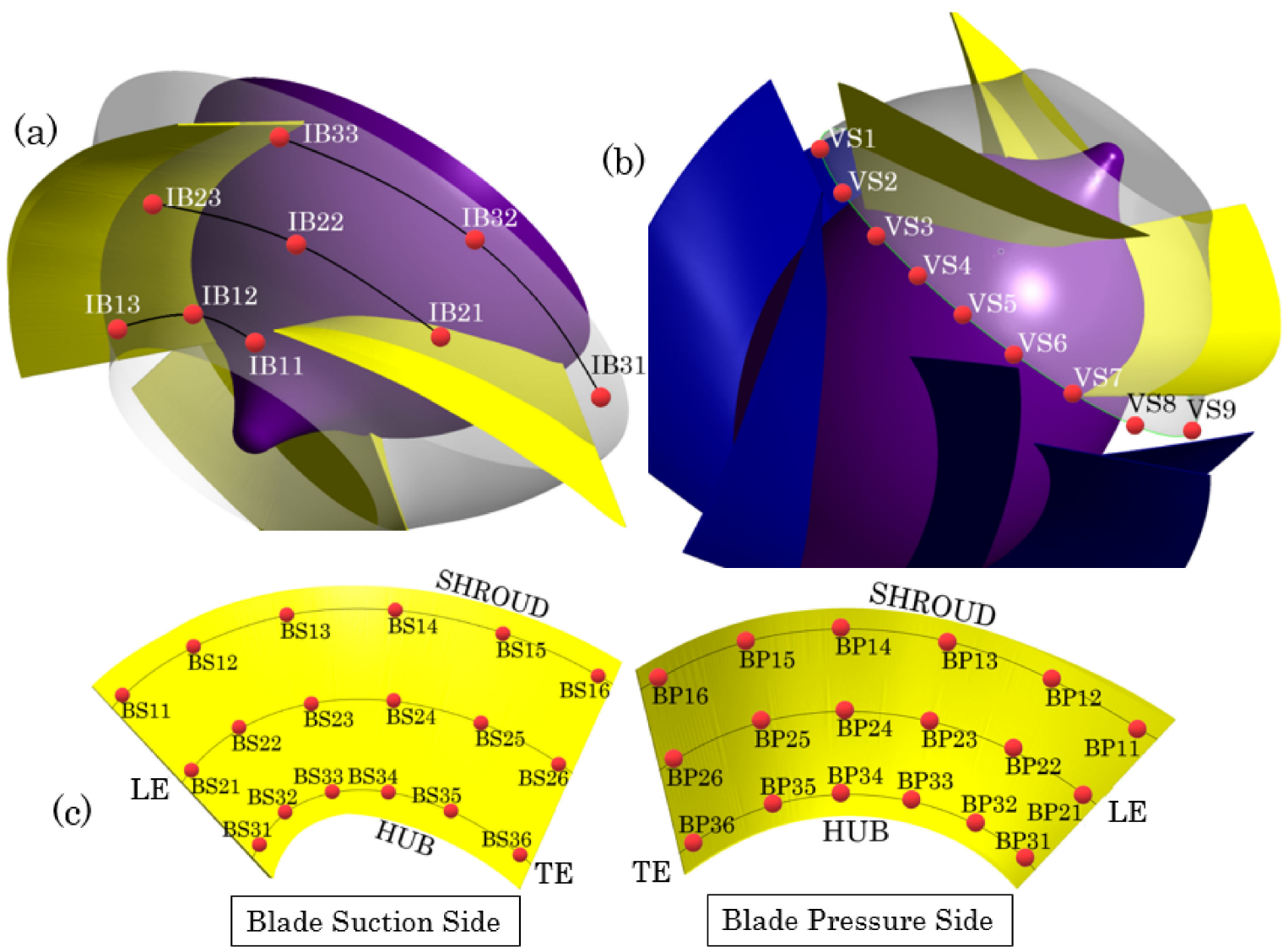

Figure 16. Pressure monitoring locations within the runner flow zone: (a) inter-blade mid-span flow zone (b) vaneless space flow zone, and (c) runner blade's suction and pressure sides.

This aspect agrees well the flow field characteristics evolution discussed above, where flow structures became more chaotic as the machine influx decreased. Under rated conditions, the associated pressure pulsation spectrum is mainly composed of three frequency components, namely the runner rotational frequency $(f n)$, the dominant frequency $f i=7 f n$, and its double (2fi). Note that the runner rotational speed is $150 \mathrm{rpm}$, which means that $f n=2.5 \mathrm{~Hz}$, while the blade passing frequency (BPF) should be $7.5 \mathrm{~Hz}$ as the utilized runner has three blades. Moreover, while the BPF stands for perturbations of the distributor flow field by the continuously passing runner blades, the guide vane passing frequency (GPF) represents the effect of stator guide vanes on the rotor flow field. Both BPF and GPF represent the mutual interactions between the runner and distributor, which is widely known as the "Rotor-Stator Interactions" or RSI. This phenomenon is widely recognized as the powerful trigger of large pressure pulsations within hydraulic machinery, which under serious cases may result in the emergence of severe structural vibrations and subsequent machine components breakage or cracks formation. On the grounds that the investigated PAT's distributor is composed of seven guide vanes, the above-mentioned dominant frequency component $f i$ is therefore of GPF type. When the machine flow conditions shifted from rated to $0.92 Q_{B E P}$ conditions, the amplitude of the dominant frequency component fi decreases to almost $80 \%$, while two new low-frequency components (LFCs) emerged, namely $0.25 f i$ and $0.1 f i$, while the $f n$ component vanished. 


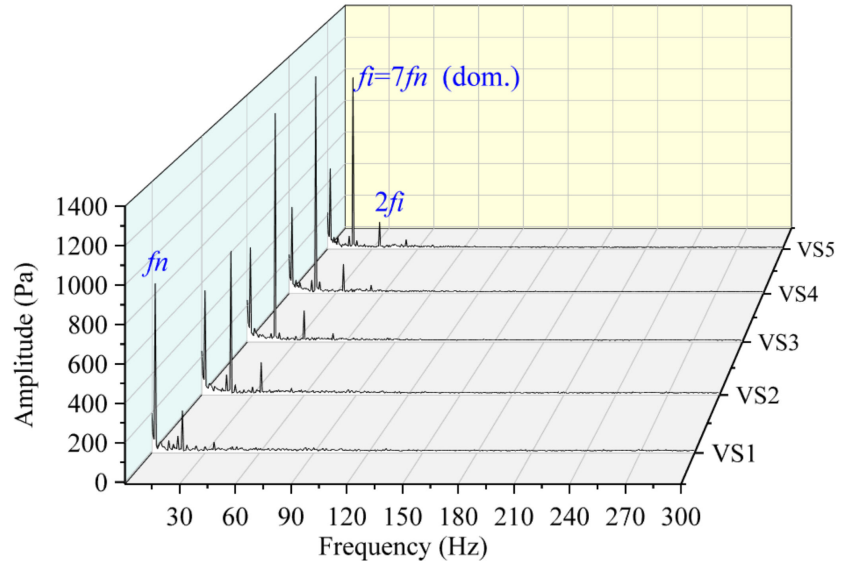

(a) 1 QвеP

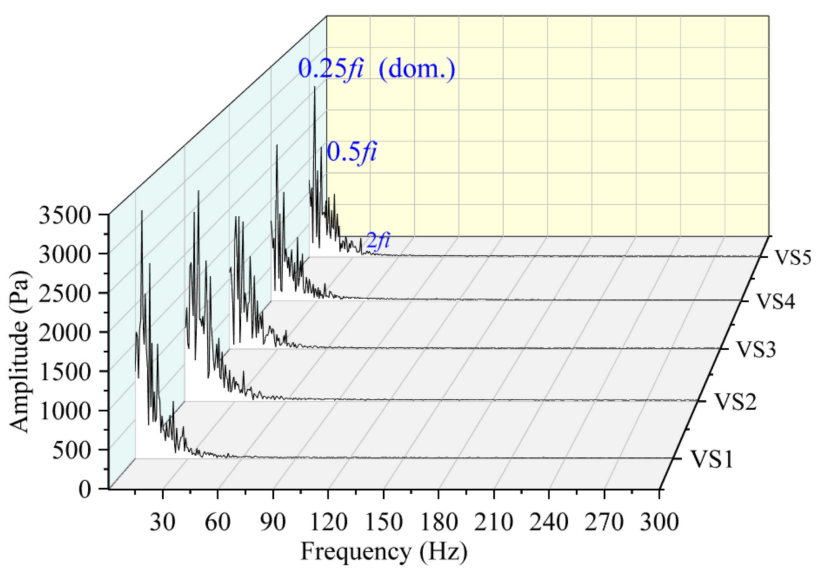

(c) $0.83 \mathrm{QBEP}$

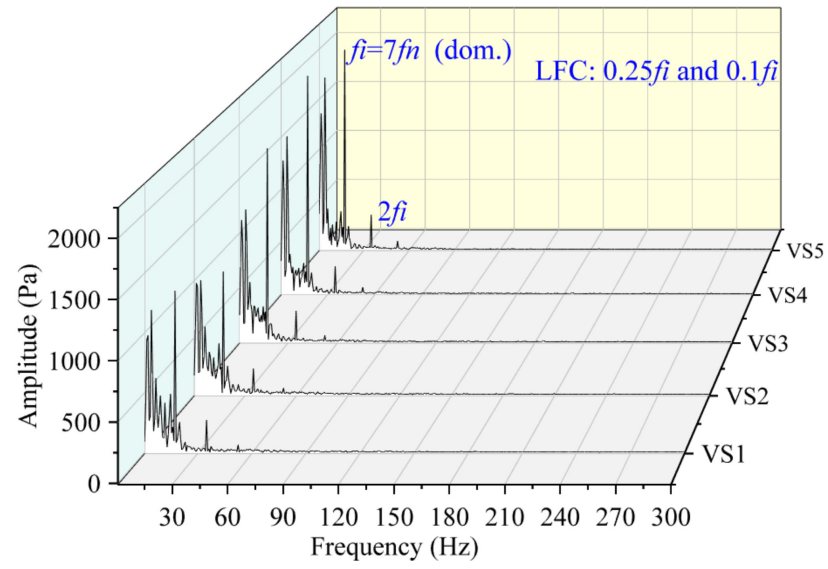

(b) 0.92 Q

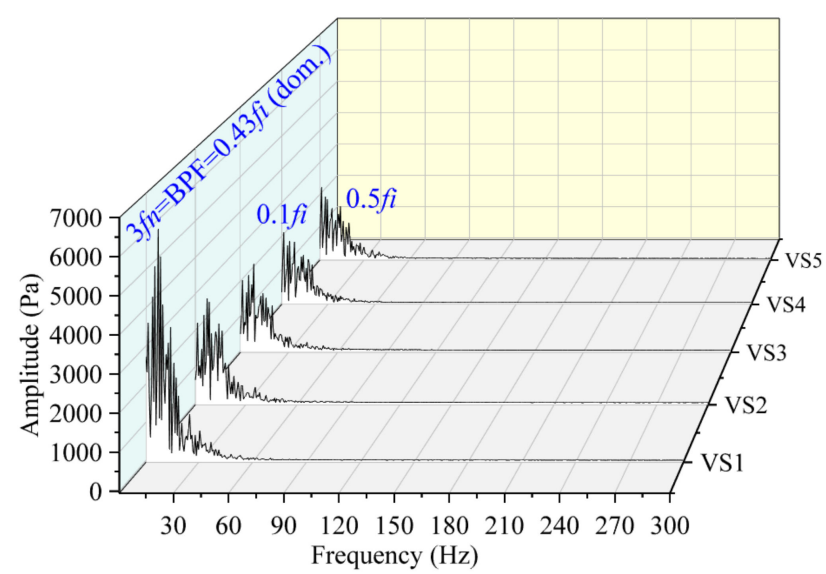

(d) 0.65 QвеP

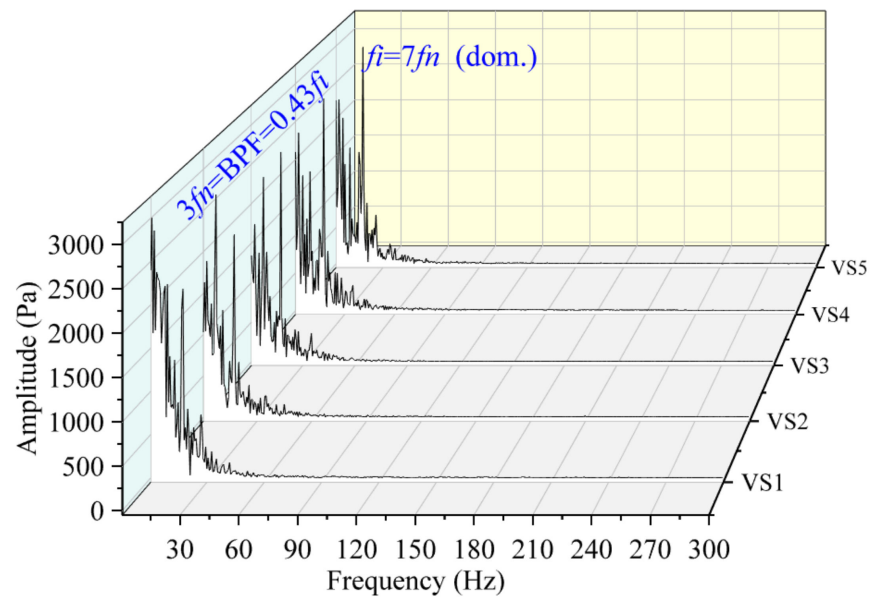

(e) 0.41 QвеР

Figure 17. Frequency domain spectra of VS pressure pulsation under different flow conditions.

The emergence of LFCs is connected to the appearance of more secondary flows as the machine's operating conditions move away from its nominal operating conditions. Under $0.83 \mathrm{Q}_{\mathrm{BEP}}$ conditions, one of the above-mentioned LFCs $(0.25 \mathrm{fi})$ became the dominant frequency, where more components in the range from $0.5 f i$ to $2 f i$ surfaced. The $0.65 Q_{B E P}$ condition presented the highest pulsation amplitude, which was exhibited by the newly emerged blade passing frequency (BPF or $3 f n$ ) component, before falling back to almost 
half its value under deep part-load conditions where the formerly dominating component fi retook the lead.

Generally speaking, pressure pulsation spectra were loaded with more low-amplitude frequency components (LAFCs) as the machine flow conditions became further from the optimum conditions, whereas the last three operating conditions stand out in this respect. As already mentioned, the emergence of these frequencies and the continuous increase in global pressure pulsation amplitudes are a result of the eventual worsening of flow unsteadiness within the machine flow zones. For the last two operating conditions in particular, the associated pressure pulsation spectra are composed of both the local flow unsteadiness-born components and the RSI-born components (BPF and GPF components).

To try to comparatively get a picture of how machine operating conditions affect the pressure pulsation levels of specific flow zones, a relative pressure pulsation coefficient $C_{P}$ was utilized, and its expression is written as in Equation (7), where $n, P_{i \max }$, and $P_{i \text { min }}$ stand for number monitors in the same location and individual point's maximum and minimum pressure pulsations amplitudes, respectively.

$$
C_{P}=\frac{1}{n \rho g H} \sum_{i=1}^{n}\left(P_{i \max }-P_{i \min }\right)
$$

In this respect, Figure 18 presents the pressure pulsation amplitude distribution mode at different locations within the runner under the five investigated operating conditions. Selected zones are the runner inter-blade channel, the vaneless space between the runner and the distributor, and the runner blade pressure and suction surfaces. Regardless of the considered operating conditions, pressure pulsation amplitudes within the inter-blade flow channels are generally found to increase in the streamwise direction from the runner inlet to the outlet. Moreover, as shown again in Figure 18a, pressure pulsation amplitudes within the inter-blade flow channels are found to globally increase with the decreasing machine flow discharge, leading to the rated conditions presenting the least of the pressure pulsation levels, while deep part-load conditions exhibited the highest pulsation amplitudes. On the other hand, for the vaneless space zone (Figure 18b), having generally exhibited the highest level of pulsation amplitudes as compared to other runner flow zones, part-load operating conditions recorded the highest amplitudes, while the least still had the rated conditions.

This is to say that, as also presented through Figure 17, vaneless space pressure pulsation amplitudes increased with the decrease in machine flow discharge, reaching their peak under part-load conditions $\left(0.65 \mathrm{Q}_{\mathrm{BEP}}\right)$ before dropping again towards deep part-load conditions. The VS pressure pulsation amplitudes distribution is more symmetric under both rated and upper part-load conditions ( $1 \mathrm{Q}_{\mathrm{BEP}}$ and $\left.0.92 \mathrm{Q}_{\mathrm{BEP}}\right)$, whereas it becomes obviously asymmetric for the last three operating conditions. This aligns well with the emergence of many LAFCs in the vaneless space pressure pulsation frequency spectra, where they were believed to be linked to the worsening of flow unsteadiness under the last three conditions.

Pressure pulsation variation mode on the runner blade's suction and pressure sides is presented in Figure 18c,d respectively. While BS1 (BP1 on the pressure side) is the closest layer to the runner shroud, BS3 (BP3 on the pressure side) is the closest layer to the hub. Pulsation amplitudes on blades' both surfaces are generally found to continuously increase with the decrease in machine flow discharge. The highest pressure pulsation amplitudes on the BSS were recorded in the runner shroud zone and vicinities under deep part-load conditions, and they decreased in the spanwise direction towards the runner hub region. The randomness in pulsation amplitudes distribution on BSS for other flow conditions is linked with the high flow activity noted at the same zone. On the other hand, the BPS's lowest pressure pulsation amplitudes were recorded within the runner shroud zone and vicinities under deep part-load conditions and increased in the spanwise direction downwards to the hub zone. Unlike in the BSS case, this trend was maintained for the entire range of investigated flow conditions. Pressure pulsation distribution mode on both of the blade's surfaces depends on frequent positions of flow vortical structures within 
this area. As also shown in Figure 11, flow vortice formation and activity were seen at the BSS's shroud zone, which was classified as type B. On the other hand, type C on BPS's shroud zone was observed only under deep part-load conditions and vanished for other flow conditions. This somehow correlates with the above-mentioned fact that BSS recorded the highest pulsation amplitudes in shroud vicinities, while it was the hub zone for BPS.

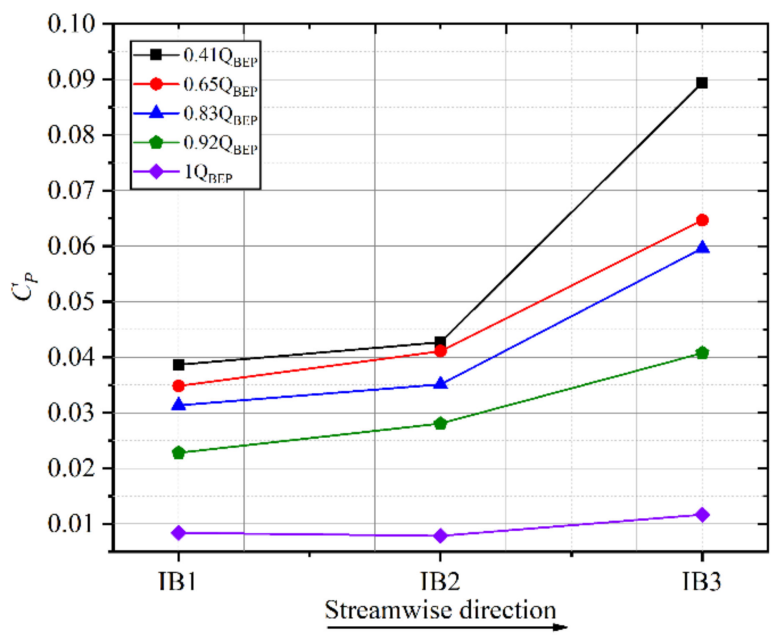

(a) IB zone

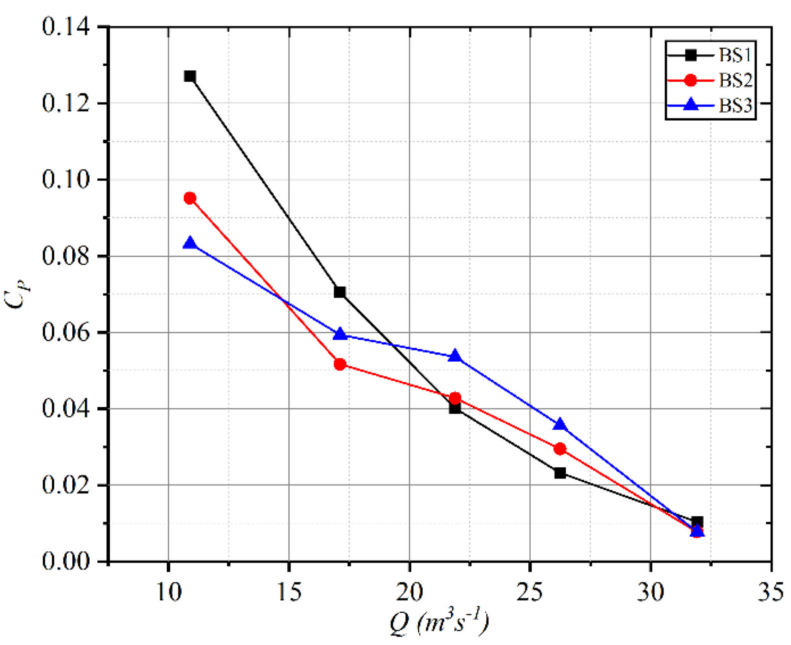

(c) BSS

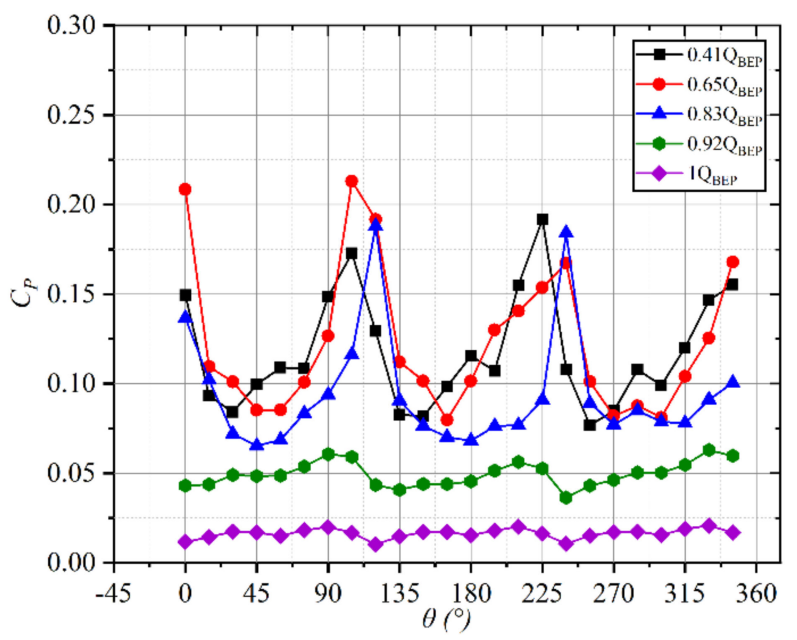

(b) VS zone

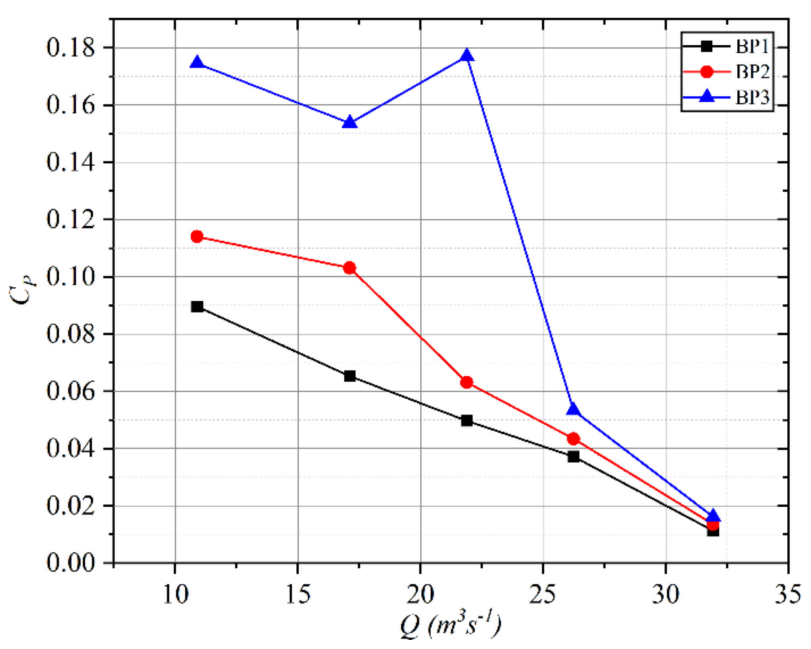

(d) BPS

Figure 18. Pressure pulsation amplitude variation within the runner and vaneless space flow zones.

\subsection{Effect of Runner Blade Angle}

Having investigated PAT flow structures formation mechanism and associated pressure pulsation characteristics, as well as their distribution modes for different operating conditions, the present study has also tried to investigate the effect of runner blade geometric design on them. In this respect, the runner blade angle $\gamma$ was selected as the testing parameter, and its three values, namely $\gamma_{1}=-2^{\circ}, \gamma_{2}=0^{\circ}$, and $\gamma_{3}=+2^{\circ}$, were selected for further analysis. Investigation on incurred changes in PAT flow dynamics with the varying blade angle was conducted where only two flow conditions, namely the deep-part-load and rated conditions, were studied.

Investigations on incurred changes in PAT flow dynamics with the varying blade angle were conducted where only two flow conditions, namely the deep-part-load (0.41 $\left.\mathrm{Q}_{\mathrm{BEP}}\right)$ and rated conditions $\left(1 \mathrm{Q}_{\mathrm{BEP}}\right)$, were studied. 
In Figure 19, streamlines on the blade's suction, colored according to velocity, and pressure surfaces for the three blade angles, namely $\gamma_{1}=-2^{\circ}, \gamma_{2}=0^{\circ}$, and $\gamma_{3}=2^{\circ}$, under deep part-load conditions, are shown. It is obvious that the change in runner blade angle altered the flow structures formation mechanism on both surfaces, where, for instance, the formerly mentioned BSS flow vortices at runner shroud and vicinal zones were comparatively weak when using the $-2^{\circ}$ blade angle. These vortices were formerly classified as type B (see Figure 11). However, with the blade angle increase from $-2^{\circ}$ through $0^{\circ}$ to $2^{\circ}$, these vortices eventually became wider, occupying more space and becoming stronger. In addition, the high-speed flow zone at the runner blade's leading edge grew wider with the increase in runner blade angle. Associated flow separation in the form of wakes was classified as type A in Figure 11. On the other hand, the BPS flow field saw the runner hub-attached vortex flow grow stronger as the blade angle increased. These flow vortices within the hub vicinities are the reason why BPS's hub pressure pulsation amplitudes were the highest as compared to other locations along the spanwise direction, as shown in Figure 18d. These vortices, partly linking to VS flow unsteadiness within the blade trailing edge vicinal zones, caused the flow reversal movement back to inter-blade channels, where the corresponding hub backflow zone grew wider with the increase in runner blade angle. To support these facts, Figures 20 and 21 give more details about the runner flow and pressure field changes taking place as the runner blade angle increases. In Figure 20, pressure distribution mode on both BSS and BPS and eventual changes as the blade angle increased are presented. As shown in this figure, and in line with the presented details presented above, the BLE low-pressure zone on the BSS corresponding to type A flow separation zone grew wider with the blade angle increase. Moreover, on the BPS, two high pressure zones can be seen, both the shroud and hub zones, corresponding to type $\mathrm{C}$ vortex flow and hub backflow zones, respectively. Both high-pressure zones are seen to increase in size and strength as the blade angle increased. In line with the above, flow axial velocity contours within the vicinal flow zone of the runner outlet are shown in Figure 21. At this zone, two high-velocity zones can be commonly found at each of the three investigated blade angles. The first is attached on the BSS, where it extends from the hub to over mid-span zone but does not reach the shroud side. The second is attached to the shroud and extends from the first blade's pressure side vicinal zone towards the next blade's suction side but does not exactly reach it. Both zones are globally found to become thicker and stronger with the blade angle increase. In addition, one low axial velocity zone (negative axial velocity or backflow) is commonly found at each of the three investigated blade angles.

This zone is attached to the hub and is detached from the BPS in the first place, but extends obliquely towards the BPS to finally touch it at the mid-span's vicinal zone. This zone is finally found to grow in size, occupying more area both at the hub and on the BPS, where it reaches the mid-span zone under $\gamma=0^{\circ}$ conditions and goes beyond for $\gamma=+2^{\circ}$. This agrees well with the details presented above, where it was stated that the hub-attached backflow zone grows with the blade angle increase. This also explains the increase in shroud-attached high axial flow zone strengthening with blade angle increase. As the hub-attached backflow zone thickens, the corresponding flow blockage pushes more water flow towards the shroud zone. Because of the axis channel flow obstruction, the shroud-attached positive flow accelerates more and more with the increase in hub backflow zone. In the same respect, the noted negative flow zone in the vicinal zone of the BSS towards the shroud side under $\gamma=0^{\circ}$ conditions (Figure 21a) also caused the flow blockage at the same zone, which led to the emergence of shroud-attached accelerated water flow zone that touches the BSS on its shroud tip region. This zone is, however, found to disappear with the increase in runner blade angle. 


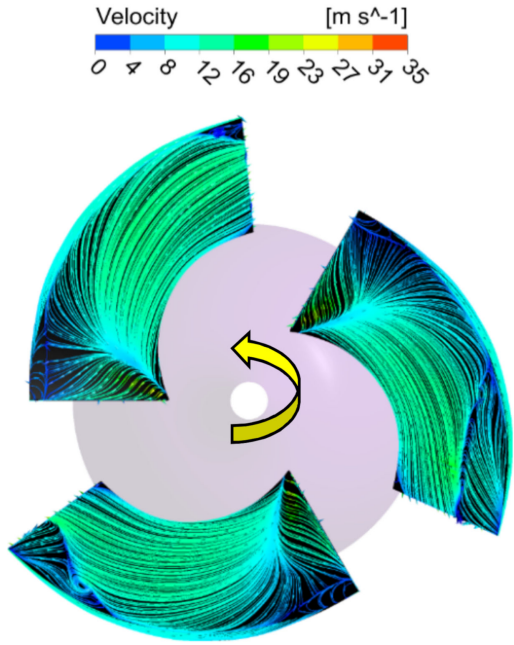

(a) $0.41 \mathrm{QBEP}\left(\gamma=-2^{\circ}\right)$

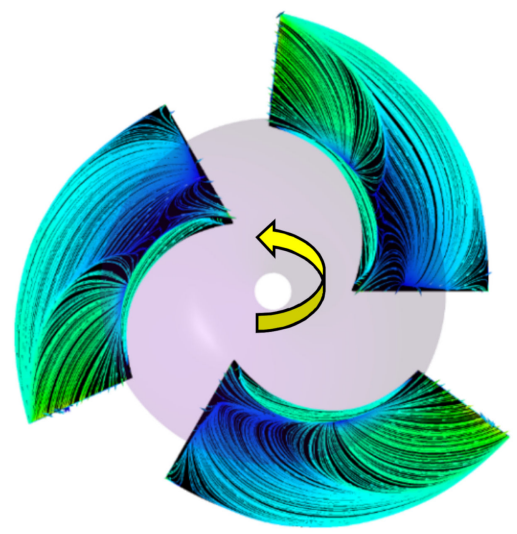

(a) $0.41 \mathrm{QBEP}\left(\gamma=-2^{\circ}\right)$

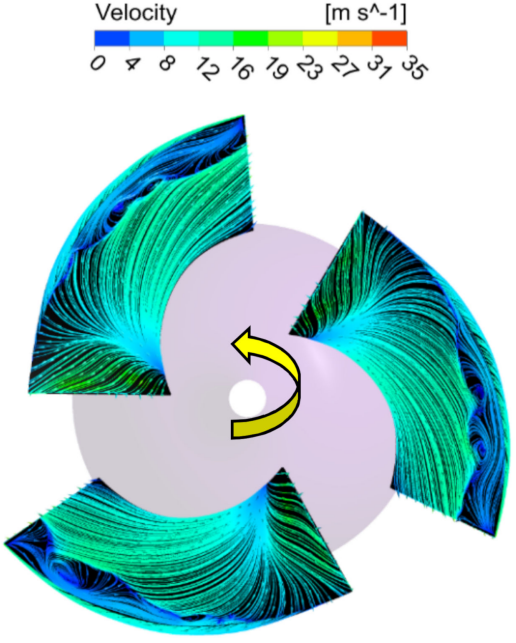

(b) $0.41 \operatorname{QBEP}\left(\gamma=0^{\circ}\right)$

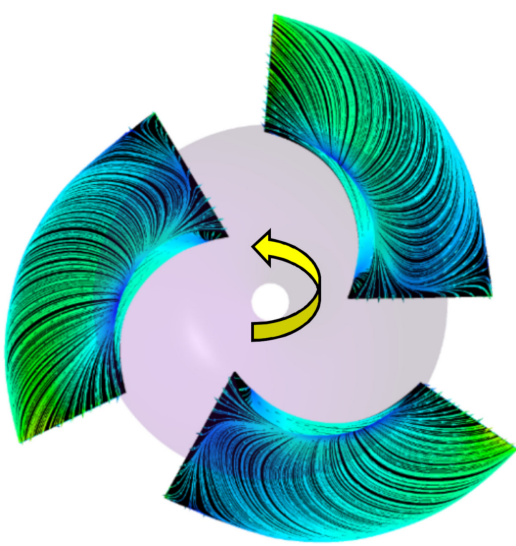

(b) $0.41 \mathrm{Q}_{\mathrm{BEP}}\left(\gamma=0^{\circ}\right)$

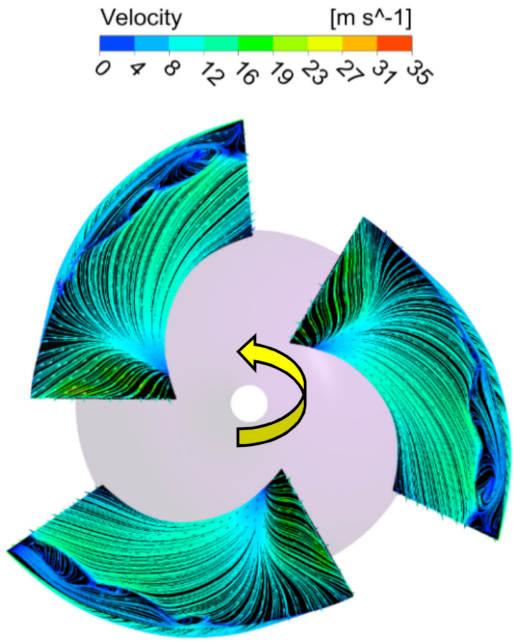

(c) $0.41 \mathrm{QBEP}\left(\gamma=+2^{\circ}\right)$

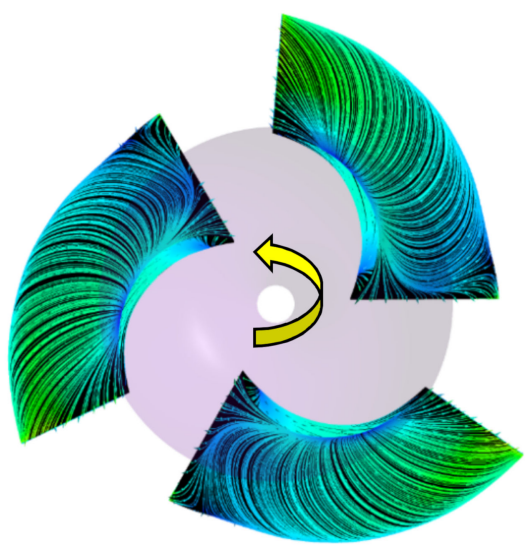

(c) $0.41 \mathrm{Q} \operatorname{BEP}\left(\gamma=+2^{\circ}\right)$

Figure 19. Streamlines colored according to velocity on runner blade's suction (up) and pressure surfaces (down) under deep part-load flow conditions $\left(0.41 \mathrm{Q}_{\mathrm{BEP}}\right)$ for different blade angles.
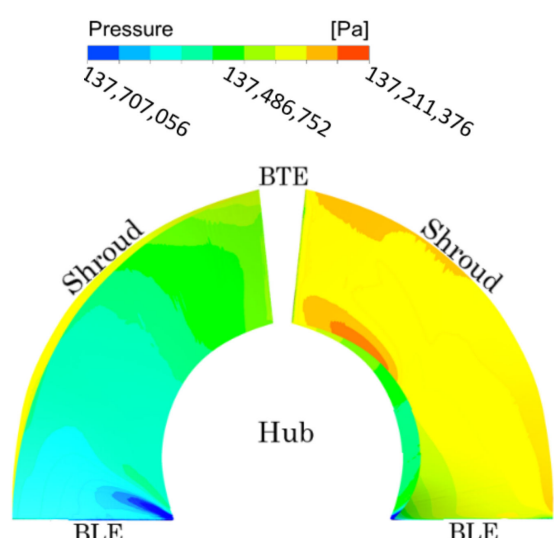

(a) $0.41 \mathrm{QBEP}\left(\gamma=-2^{\circ}\right)$
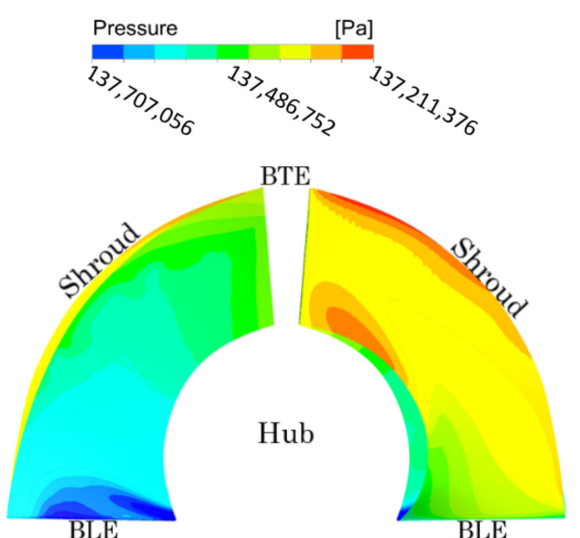

(b) $0.41 \mathrm{QBEP}\left(\gamma=0^{\circ}\right)$
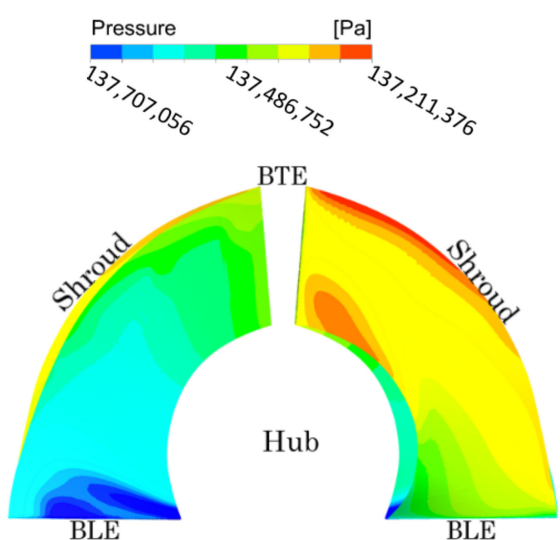

(c) $0.41 \mathrm{QBEP}\left(\gamma=+2^{\circ}\right)$

Figure 20. Pressure distribution contours on runner BSS (left side of each figure) and BPS (right side of each figure) under deep part-load conditions (0.41 QBEP). 


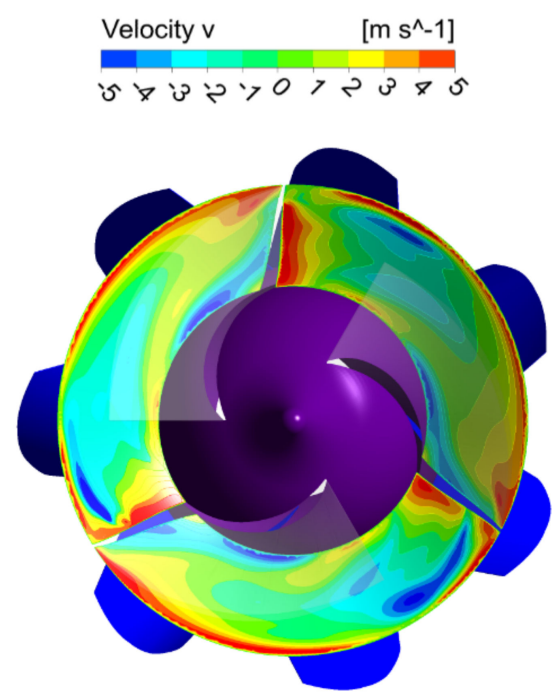

(a) $0.41 \operatorname{QBEP}\left(\gamma=-2^{\circ}\right)$

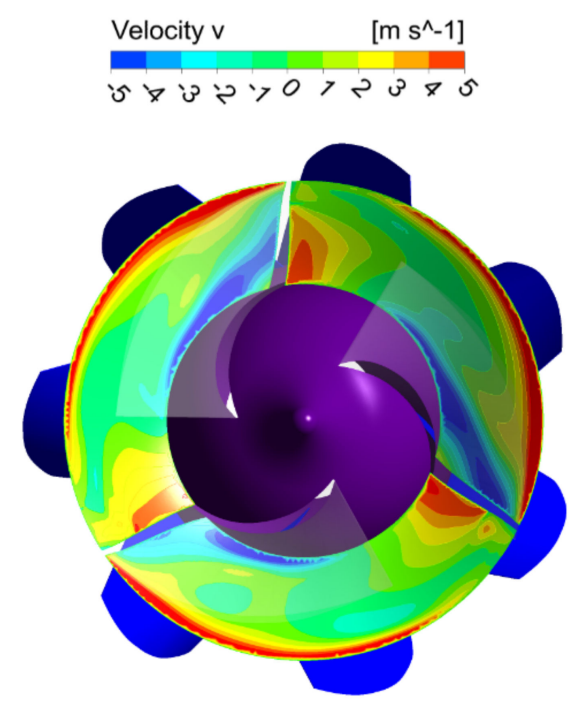

(b) $0.41 \operatorname{QBEP}\left(\gamma=0^{\circ}\right)$

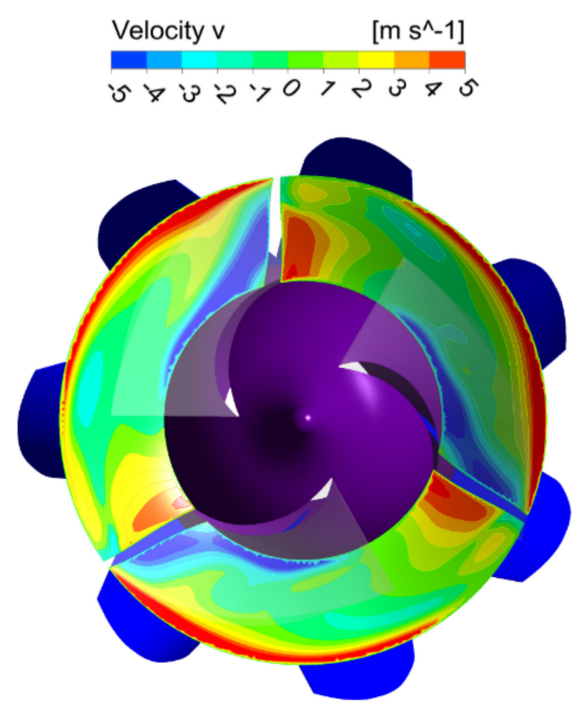

(c) $0.41 \mathrm{QBEP}\left(\gamma=+2^{\circ}\right)$

Figure 21. Axial velocity contours at the runner outlet for the three blade angles under deep part-load conditions ( $\left.0.41 \mathrm{Q}_{\mathrm{BEP}}\right)$.

Having described PAT flow dynamics and eventual changes as the blade angle increased, an effort was also made to explore corresponding modifications of pressure pulsation characteristics and distribution mode at different components of the considered computational domain. In this respect, while considering two operating conditions, namely the deep part-load and rated conditions, Figure 22 displays the pressure pulsation amplitudes distribution mode within the vaneless space flow zone for the three investigated blade angles. The pressure pulsation level of deep part-load conditions is globally found to be almost four times higher than the rated conditions. Having considered 24 monitoring locations equidistantly positioned along the VS circumference on the mid-span plane, the pulsation amplitude is found to increase with the blade angle under the rated conditions, while the situation is complex for deep part-load conditions, where pulsation amplitudes at different locations almost randomly vary, following no common law. Nevertheless, the highest of VS pulsation amplitudes under deep part-load condition was recorded under $\gamma=0^{\circ}$ blade angle at VS16 location. In Figure 23, streamwise pressure pulsation amplitudes distribution mode from the inlet pipe (IP) through inter-blade and inter-guide vane channels (IB and IG) to the outlet pipe (OP), considering the three-blade angles, is presented. Four axially equidistant monitoring locations expanding from the IP's elbow zone to the runner inlet were selected as IP4, IP3, IP2, and IP1. Within the inter-blade zone, three monitoring locations, namely IB12, IB22, and IB32, were selected in the streamwise direction from the runner inlet to the outlet's vicinal zone on the runner's mid-span plane. Again, considering the distributor's mid-span plane, four successive locations were selected in the streamwise direction from the inlet to the outlet's vicinal as IG21, IG22, IG23, and IG24. Finally, starting from the OP's inlet zone to its elbow zone, four more equidistant monitoring locations were selected as OP11, OP21, OP31, and OP4. Therefore, adding the VS monitoring location, a total of 16 pressure monitoring locations were chosen all along the flow passage route from the IP's elbow zone to the OP's elbow zone. As shown in Figure 23, regardless of the considered blade angle or operating condition, pulsation amplitudes within the IP zone are low and almost constant, followed by a gradual rise through the runner IB channels and vaneless space, reaching the highest level of pulsation amplitudes within the inter-guide vanes channels before falling back to the OP zone. Under the rated conditions, the PAT model with $\gamma=+2^{\circ}$ recorded the highest of the pressure pulsation amplitudes at most of the flow zone along the investigated range, followed by $\gamma=0^{\circ}$ and finally $\gamma=-2^{\circ}$, presenting the lowest pressure pulsation level. Under deep part-load conditions on the other side, $\gamma=-2^{\circ}$ dominated the whole flow passage from IP elbow through runner IB zone to the VS zone, mostly followed by 
$\gamma=0^{\circ}$. Within the distributor's IG channels, $\gamma=+2^{\circ}$ took the lead again, followed again by $\gamma=0^{\circ}$ for the majority of locations. As for the OP flow zone, $\gamma=0^{\circ}$ dominated, followed by $\gamma=+2^{\circ}$ and $\gamma=-2^{\circ}$, respectively. Moreover, Figure 23b gives more clarity to some aspects presented in Figure 22b. Under deep part-load conditions, the averaged values of recorded pulsation amplitudes within the VS zone show that the highest of the pulsation amplitudes under these conditions were recorded with the $\gamma=-2^{\circ}$ followed by $\gamma=0^{\circ}$ and finally $\gamma=+2^{\circ}$. This, therefore, represents an opposite situation as compared to the rated conditions situation. However, on a global scale, regardless of the machine operating conditions, the distributor inter-guide vanes flow zone recorded the highest of pressure pulsation amplitudes, where, among the three investigated blade angles, the PAT model with $\gamma=+2^{\circ}$ blade angle exhibited the highest of pressure pulsation levels, followed by $\gamma=0^{\circ}$ and $\gamma=-2^{\circ}$, respectively.

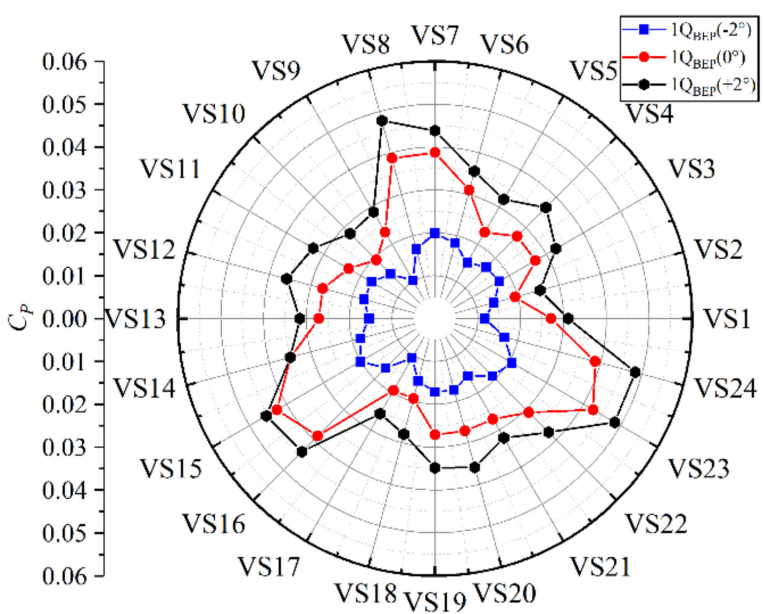

(a)

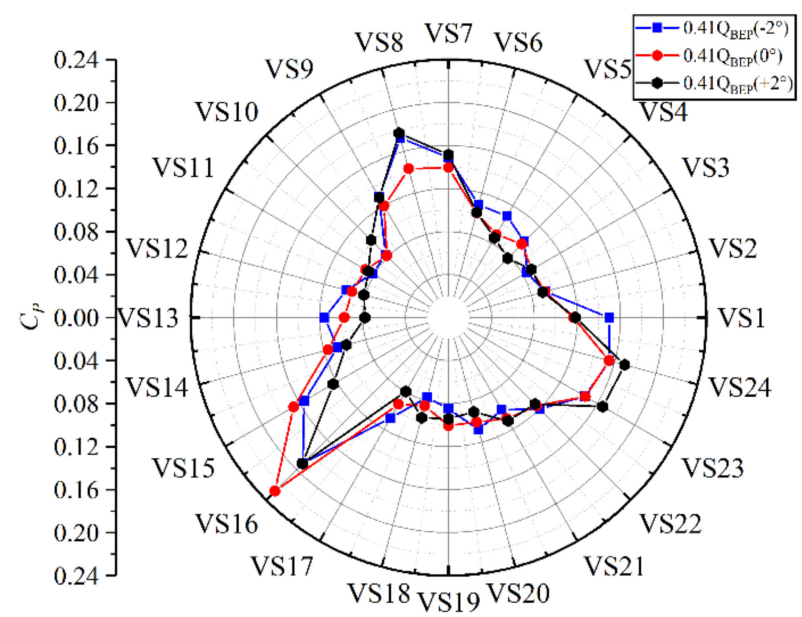

(b)

Figure 22. VS pressure pulsation distribution mode for three runner blade angles: (a) rated operating condition, (b) deep part-load operating condition.

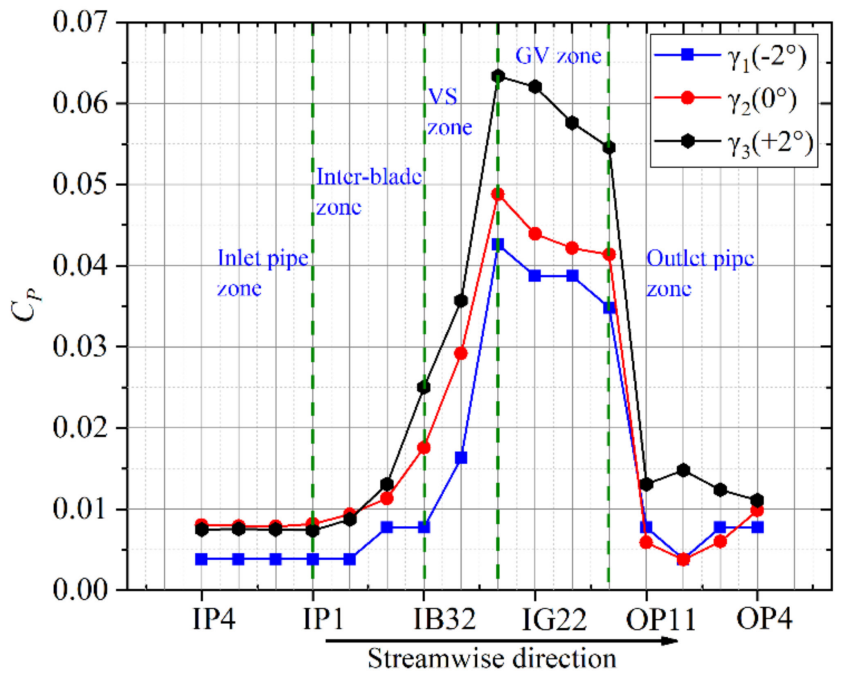

(a)

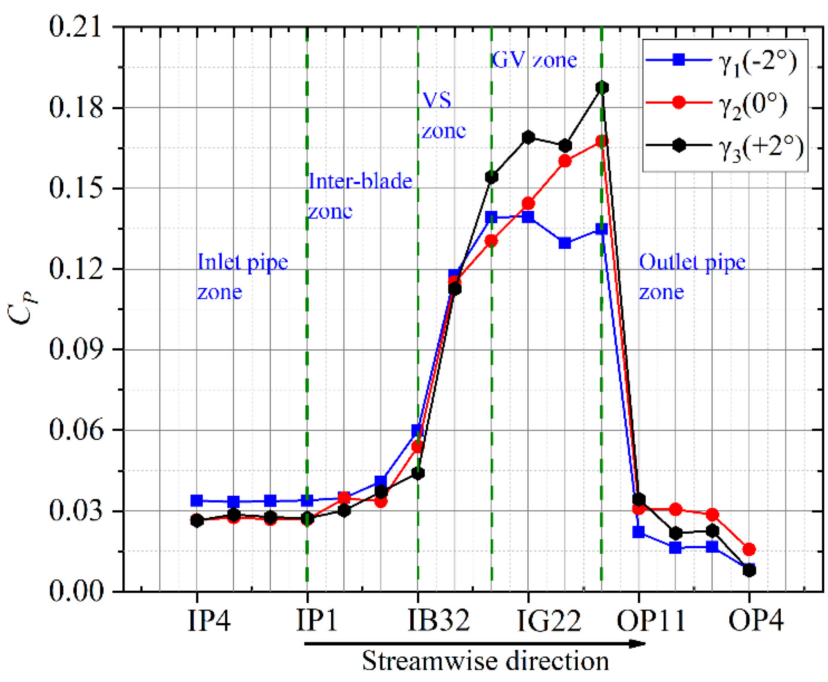

(b)

Figure 23. Pressure pulsation distribution in the streamwise direction for different $\gamma$ values: (a) rated operating condition, (b) deep part-load operating condition. 


\section{Conclusions}

In this study, experimental and numerical simulations were carried out to investigate the performance characteristics of a mixed-flow PAT under pump mode, as well as the associated flow structure formation mechanism for different operating conditions and runner blade angles. The investigated PAT model does not have the blade tip clearance $(\delta=0)$, considering five operating conditions, ranging between the optimum and deep part-load conditions, and three blade angles, namely $-2^{\circ}, 0^{\circ}$, and $+2^{\circ}$. The following conclusions have been drawn.

Within the decrease in machine flow from optimum conditions to deep part-load conditions, flow unsteadiness gradually becomes more serious, where the last three conditions (0.41 Q $\mathrm{Q}_{\mathrm{BEP}}, 0.65 \mathrm{Q}_{\mathrm{BEP}}$, and $0.83 \mathrm{Q}_{\mathrm{BEP}}$ ) presented the most disturbed flow structures. Under these conditions, the most dominated regions by flow vortical structures within the runner are runner blade leading edge, the BSS's shroud side, the BPS's hub side, and the runner outlet zone, while for the distributor component, the hub zone and GPS were the ones that experienced the largest flow unsteadiness.

Pressure pulsation amplitudes were found to increase with the decreasing machine flow discharge, reaching its peak under part-load conditions $\left(0.65 \mathrm{Q}_{\mathrm{BEP}}\right)$ before falling back to deep part-load conditions. Depending on the considered operating conditions, the vaneless space pressure pulsation spectra have been found to be composed of RSI-born frequency components (guide vane passing frequency and blade passing frequency) and local flow unsteadiness-borne LAFCs. The RSI-associated components served the dominant frequency components for the majority of investigated operating conditions.

The runner blade angle caused changes in the machine flow and pressure field characteristics, where, for instance, with the increase of runner blade angle from $-2^{\circ}$ through $0^{\circ}$ to $2^{\circ}$, the runner flow unsteadiness only correspondingly worsened. Pressure pulsation levels within the vaneless space flow zone were found to increase with the blade angle under optimum conditions, while the opposite occurred under deep part-load conditions. However, on a global scale, the highest pulsation amplitudes were recorded within the distributor components, and they increased with the runner blade angle.

Now that the PAT model's pump mode flow dynamics for a wide range of flows and three blade angles have been studied, the next research focus will turn to investigating the same aspects considering the turbine mode of operation. An attempt will be made to establish a relationship between flow structures formation mechanisms of PAT's turbine and pumping modes. Moreover, an optimization scheme will be utilized to improve both modes' flow field characteristics, leading to reasonably higher performances in both modes.

Author Contributions: Conceptualization, M.B., K.K. and H.C.; Methodology, M.B., H.C. and A.M.; Software, M.B., A.M. and G.M.B.; Formal analysis, M.B., K.K., H.C., Y.Z. and D.Z.; Writing-original draft, M.B., A.M. and G.M.B.; Writing-review\&editing, Y.Z., D.Z., H.C. and K.K.; Resources, K.K., Y.Z. and D.Z.; Supervision, K.K. and Y.Z.; Funding acquisition, M.B., K.K. and H.C. All authors have read and agreed to the published version of the manuscript.

Funding: This study was supported by the Postdoctoral Research Fund of Jiangsu Province (2020Z152), National Natural Science Foundation of China (52009033; 52006053), Natural Science Foundation of Jiangsu Province (BK20200509; BK20200508), and the Fundamental Research Funds for Central Universities (B210202066).

Acknowledgments: The support from the College of Water Conservancy and Hydropower Engineering at Hohai University, China is gratefully acknowledged. The authors would also like to acknowledge the technical support and advices from Engineer NDAYIZIGIYE Janvier of Rukarara Hydroelectric Plant (RHP), Rwanda.

Conflicts of Interest: The authors declare no conflict of interest. 


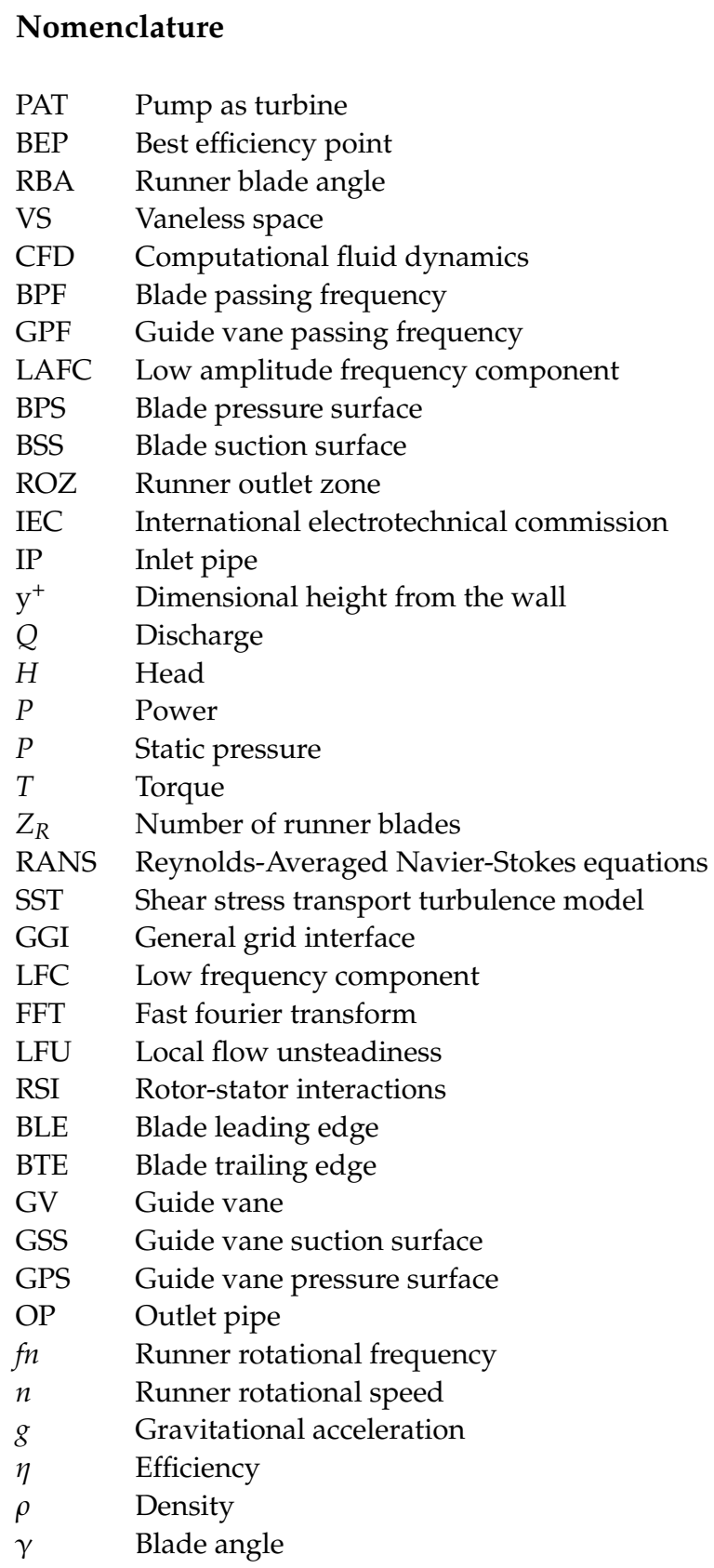

\section{References}

1. Ramos, H.; Borga, A. Pumps as turbines: An unconventional solution to energy production. Urban Water 1999, 1, $261-263$. [CrossRef]

2. Binama, M.; Su, W.-T.; Li, X.-B.; Li, F.-C.; Wei, X.-Z.; An, S. Investigation on pump as turbine (PAT) technical aspects for micro hydropower schemes: A state-of-the-art review. Renew. Sustain. Energy Rev. 2017, 79, 148-179. [CrossRef]

3. Jain, S.V.; Patel, R.N. Investigations on pump running in turbine mode: A review of the state-of-the-art. Renew. Sustain. Energy Rev. 2014, 30, 841-868. [CrossRef]

4. Venturini, M.; Alvisi, S.; Simani, S.; Manservigi, L. Energy Production by Means of Pumps As Turbines in Water Distribution Networks. Energies 2017, 10, 1666. [CrossRef]

5. Carravetta, A.; Del Giudice, G.; Fecarotta, O.; Ramos, H.M. Energy Production in Water Distribution Networks: A PAT Design Strategy. Water Resour. Manag. 2012, 26, 3947-3959. [CrossRef]

6. Zakkour, P.D.; Gaterell, M.R.; Griffin, P.; Gochin, R.J.; Lester, J.N. Developing a sustainable energy strategy for a water utility. Part II: A review of potential technologies and approaches. J. Environ. Manag. 2002, 66, 115-125. [CrossRef] [PubMed]

7. Patelis, M.; Kanakoudis, V.; Gonelas, K. Pressure Management and Energy Recovery Capabilities Using PATs. Procedia Eng. 2016, 162, 503-510. [CrossRef] 
8. Sinagra, M.; Sammartano, V.; Morreale, G.; Tucciarelli, T. A New Device for Pressure Control and Energy Recovery in Water Distribution Networks. Water 2017, 9, 309. [CrossRef]

9. De Marchis, M.; Milici, B.; Volpe, R.; Messineo, A. Energy Saving in Water Distribution Network through Pump as Turbine Generators: Economic and Environmental Analysis. Energies 2016, 9, 877. [CrossRef]

10. De Marchis, M.; Freni, G. Pump as turbine Implementation in a dynamic numerical model: Cost analysis for energy recovery in water distribution network. J. Hydroinform. 2015, 17, 347-360. [CrossRef]

11. Lugauer, F.J.; Kainz, J.; Gaderer, M. Techno-Economic Efficiency Analysis of Various Operating Strategies for Micro-Hydro Storage Using a Pump as a Turbine. Energies 2021, 14, 425. [CrossRef]

12. Carravetta, A.; Fecarotta, O.; Sinagra, M.; Tucciarelli, T. Cost-Benefit Analysis for Hydropower Production in Water Distribution Networks by a Pump as Turbine. J. Water Resour. Plan. Manag. 2014, 140, 04014002. [CrossRef]

13. Giosio, D.R.; Henderson, A.D.; Walker, J.M.; Brandner, P.A.; Sargison, J.E.; Gautam, P. Design and performance evaluation of a pump-as-turbine micro-hydro test facility with incorporated inlet flow control. Renew. Energy 2015, 78, 1-6. [CrossRef]

14. Crespo Chacón, M.; Rodríguez Díaz, J.A.; García Morillo, J.; McNabola, A. Hydropower energy recovery in irrigation networks: Validation of a methodology for flow prediction and pump as turbine selection. Renew. Energy 2020, 147, 1728-1738. [CrossRef]

15. Polák, M. The Influence of Changing Hydropower Potential on Performance Parameters of Pumps in Turbine Mode. Energies 2019, 12, 2103. [CrossRef]

16. Mauro, V.; Stefano, A.; Silvio, S.; Lucrezia, M.J.E. Comparison of Different Approaches to Predict the Performance of Pumps As Turbines (PATs). Energies 2018, 11, 1016. [CrossRef]

17. Frosina, E.; Buono, D.; Senatore, A. A Performance Prediction Method for Pumps as Turbines (PAT) Using a Computational Fluid Dynamics (CFD) Modeling Approach. Energies 2017, 10, 103. [CrossRef]

18. Barbarelli, S.; Amelio, M.; Florio, G. Predictive model estimating the performances of centrifugal pumps used as turbines. Energy 2016, 107, 103-121. [CrossRef]

19. Barbarelli, S.; Amelio, M.; Florio, G.; Scornaienchi, N.M. Procedure Selecting Pumps Running as Turbines in Micro Hydro Plants. Energy Procedia 2017, 126, 549-556. [CrossRef]

20. Amelio, M.; Barbarelli, S.; Schinello, D. Review of Methods Used for Selecting Pumps as Turbines (PATs) and Predicting Their Characteristic Curves. Energies 2020, 13, 6341. [CrossRef]

21. Renzi, M.; Nigro, A.; Rossi, M. A methodology to forecast the main non-dimensional performance parameters of pumps-asturbines (PaTs) operating at Best Efficiency Point (BEP). Renew. Energy 2020, 160, 16-25. [CrossRef]

22. Balacco, G. Performance Prediction of a Pump as Turbine: Sensitivity Analysis Based on Artificial Neural Networks and Evolutionary Polynomial Regression. Energies 2018, 11, 3497. [CrossRef]

23. Štefan, D.; Rossi, M.; Hudec, M.; Rudolf, P.; Nigro, A.; Renzi, M. Study of the internal flow field in a pump-as-turbine (PaT): Numerical investigation, overall performance prediction model and velocity vector analysis. Renew. Energy 2020, 156, 158-172. [CrossRef]

24. Shen, S.; Qian, Z.; Ji, B. Numerical Analysis of Mechanical Energy Dissipation for an Axial-Flow Pump Based on Entropy Generation Theory. Energies 2019, 12, 4162. [CrossRef]

25. Ghorani, M.M.; Sotoude Haghighi, M.H.; Maleki, A.; Riasi, A. A numerical study on mechanisms of energy dissipation in a pump as turbine (PAT) using entropy generation theory. Renew. Energy 2020, 162, 1036-1053. [CrossRef]

26. Lin, T.; Li, X.; Zhu, Z.; Xie, J.; Li, Y.; Yang, H. Application of enstrophy dissipation to analyze energy loss in a centrifugal pump as turbine. Renew. Energy 2021, 163, 41-55. [CrossRef]

27. Ntiri Asomani, S.; Yuan, J.; Wang, L.; Appiah, D.; Adu-Poku, K.A. The Impact of Surrogate Models on the Multi-Objective Optimization of Pump-As-Turbine (PAT). Energies 2020, 13, 2271. [CrossRef]

28. Wang, L.; Asomani, S.N.; Yuan, J.; Appiah, D. Geometrical Optimization of Pump-As-Turbine (PAT) Impellers for Enhancing Energy Efficiency with 1-D Theory. Energies 2020, 13, 4120. [CrossRef]

29. Wang, T.; Kong, F.; Xia, B.; Bai, Y.; Wang, C. The method for determining blade inlet angle of special impeller using in turbine mode of centrifugal pump as turbine. Renew. Energy 2017, 109, 518-528. [CrossRef]

30. Hongyu, G.; Wei, J.; Yuchuan, W.; Hui, T.; Ting, L.; Diyi, C. Numerical simulation and experimental investigation on the influence of the clocking effect on the hydraulic performance of the centrifugal pump as turbine. Renew. Energy 2021, 168, 21-30. [CrossRef]

31. Tahani, M.; Kandi, A.; Moghimi, M.; Houreh, S.D. Rotational speed variation assessment of centrifugal pump-as-turbine as an energy utilization device under water distribution network condition. Energy 2020, 213, 118502. [CrossRef]

32. Han, Y.; Tan, L. Influence of rotating speed on tip leakage vortex in a mixed flow pump as turbine at pump mode. Renew. Energy 2020, 162, 144-150. [CrossRef]

33. Liu, Y.; Han, Y.; Tan, L.; Wang, Y. Blade rotation angle on energy performance and tip leakage vortex in a mixed flow pump as turbine at pump mode. Energy 2020, 206, 118084. [CrossRef]

34. Ji, L.; Li, W.; Shi, W.; Chang, H.; Yang, Z. Energy characteristics of mixed-flow pump under different tip clearances based on entropy production analysis. Energy 2020, 199, 117447. [CrossRef]

35. IEC. Hydraulic Turbines, Storage Pumps and Pump-Turbines-Model Acceptance Tests; IEC: Geneva, Switzerland, 1999; Volume IEC 60193, p. 569.

36. Menter, F.R. Two-Equation Eddy-Viscosity Turbulence Models for Engineering Applications. Aiaa J. 1994, 32, 1598-1605. [CrossRef] 
37. Menter, F.R. Review of the Shear-Stress Transport Turbulence Model Experience From an Industrial Perspective. Int. J. Comput. Fluid Dyn. 2009, 23, 305-316. [CrossRef]

38. Wilcox, D.C. Reassessment of the scale-determining equation for advanced turbulence models. Aiaa J. 1988, 26, 1299-1310. [CrossRef]

39. Jones, W.P.; Launder, B.E. The prediction of laminarization with a two-equation model of turbulence. Int. J. Heat Mass Transf. 1972, 15, 301-314. [CrossRef]

40. Woelke, M. Eddy Viscosity Turbulence Models Employed by Computational Fluid Dynamics. Trans. Inst. Aviat. 2007, Nr 4, 92-113. 